\title{
Molecular and structural basis of low interfacial energy of complex coacervates in water
}

\author{
YongSeok Jho ${ }^{1}$, Hee Young Yoo ${ }^{2}$, Yanxian Lin $^{3}$, Songi Han ${ }^{4,5}$, Dong Soo Hwang ${ }^{2,6}$ \\ ${ }^{1}$ Center for Soft and Living Matter, Institute for Basic Science (IBS), Ulsan 44919, Republic of \\ Korea \\ ${ }^{2}$ Division of Integrative Biosciences and Biotechnology, Pohang University of Science and \\ Technology (POSTECH), Pohang 37673, Republic of Korea \\ ${ }^{3}$ Biomolecular Science and Engineering, University of California Santa Barbara, Santa Barbara, \\ California 93106, USA. \\ ${ }^{4}$ Department of Chemistry and Biochemistry, University of California Santa Barbara, Santa \\ Barbara, California 93106, USA. \\ ${ }^{5}$ Department of Chemical Engineering, University of California Santa Barbara, Santa Barbara, \\ California 93106, USA. \\ ${ }^{6}$ School of Environmental Science and Engineering, Pohang University of Science and \\ Technology (POSTECH), Pohang 37673, Republic of Korea
}

\begin{abstract}
Complex coacervate refers to a phase-separated fluid, typically of two oppositely charged polyelectrolytes in solution, representing a complex fluid system that has been shown to be of essential interest to biological systems, as well as for soft materials processing owing to the expectation of superior underwater coating or adhesion properties. The significance and interest in complex coacervate fluids critically rely on its low interfacial tension with respect to water that, in turn, facilitates the wetting of macromolecular or material surfaces under aqueous conditions, provided there is attractive interaction between the polyelectrolytes constituents and the surface. However, the molecular and structural bases of these properties remain unclear. Recent studies propose that the formation of water-filled and bifluidic sponge-like nanostructured network, driven by the tuning of electrostatic interactions between the polyelectrolyte constituents or their complexes may be a common feature of complex coacervate fluids that display low fluid viscosity and low interfacial tension, but more studies are needed to verify the generality of these observations. In this review, we summarize representative studies of interfacial tension and ultrastructures of complex coacervate fluids. We highlight that a consensus property of the complex coacervate fluid is the observation of high or even bulk-like
\end{abstract}


water dynamics within the dense complex coacervate phase that is consistent with a low cohesive energy fluid. Our own studies on this subject are enabled by the application of magnetic resonance relaxometry methods relying on spin labels tethered to polyelectrolyte constituents or added as spin labeled probe molecules that partition into the dense versus the equilibrium coacervate phase, permitting the extraction of information on local polymer dynamics, polymer packing and local water dynamics. We conclude with a snapshot of our current perspective on the molecular and structural bases of the low interfacial tension of complex coacervate fluids.

\section{History of the study of complex coacervation}

Complex coacervation refers to a phenomenon describing the phase separation of a solution of two oppositely charged polyelectrolytes into two liquid phases - a dense polyelectrolyte-rich phase and a dilute polyelectrolyte-depleted phase. The dense and concentrated liquid phase is often termed the coacervate phase and the dilute phase the equilibrium phase, where the coacervate phase characteristically appears as droplets of sub- to several $\mu \mathrm{m}$ dimensions that may further coalesce to larger droplets and may be driven to macroscopic phase separation (Figure 1). Whether the coacervate phase is a homogeneous solution of polyelectrolytes and/or under what experimental conditions may display additional ultrastructures and multiple phases is an active area of interest that will be separately discussed in this review, while the minimal requirement for complex coacervation is the liquid-liquid phase separation of polyelectrolyte solutions to a polyelectrolyte-rich and a polyelectrolyte-depleted liquid as driven by the modulation of electrostatic interactions between the polyelectrolytes.

The origin and the history of the study of coacervates go back a long way to the early 1910s. If taking a very broad view, one can say that the first documented observation of this intriguing liquid-liquid separation was by Tiebackx [1] working with gelatin and gum arabic. The studies of Tiebackx had instilled tremendous interest in a group of Dutch scientists led by Bungenberg de Jong, and it was through their systematic and in-depth studies for almost two decades that they were able to define and coin the term 'coacervate'.

In order to fully distinguish the various types of coacervates, Bungenberg de Jong and his colleagues used the term complex coacervation when multiple polymer types are involved in the complexation. According to Bungenberg de Jong and his colleagues: "When oppositely charged polyelectrolytes are mixed in aqueous solution at the $\mathrm{pH}$ range where they neutralize each other, the polyelectrolytes mingle and partially desolvate to form a dynamic polymer rich fluid within a polyelectrolyte depleted fluid. This phenomenon and the resulting polymer-rich phase are defined as complex coacervation and complex coacervate, respectively. By coalescence, complex coacervates can grow from nano, micro to meso-droplets and, eventually, a dense bulk coacervate phase may separate from the solution." (Figure 1) [2]. 
In that same work, Bungenberg de Jong and his colleagues also noted an intriguing property that complex coacervate possess, namely the tendency for complex coacervates to engulf solid particles and dyes, e.g. carbon, indigo, and eosine (Figure 2, left panel). These observations are the first documented report on what is believed to be an innate physical property of complex coacervate, namely an outstanding surface wetting ability in water, which is facilitated by the low interfacial tension of the complex coacervate phase in water, with respect to water besides favorable adhesive energy between the polyelectrolytes constituents and the surface $[3,4]$.

For clarity, note that coacervation that occurs with a single polymer constituent is termed 'simple coacervation'. Simple coacearvation often relies on polyelectrolytes with multiple functional moieties included within one polymer chain that coalesces with other chains and undergo liquid-liquid phase separation to form a coacervate. Polyampholytes, a charged polymer with both positively and negatively charged groups, may form a coacervate phase that can be defined as simple coacervate [5]. Generally, proteins rich in both cationic and anionic residues should be capable of forming simple coacervates, as indeed observed with gelatin [6] and soy glycinin protein [7]. One recent example of protein that forms simple coacervation through this manner was reported in a mussel protein system [8]. Another example is LAF, a caenorhabditis elegans protein, that was found to form protein droplets by tuning salt concentration, as well as in the presence of RNA[9]. Consistent with the concept just presented, the primary protein sequence of LAF contains roughly equal numbers of cationic and anionic residues. However, whether the LAF-based protein droplet is indeed a simple coacervate phase is a question that has yet to be verified.

The process of complex coacervation is generally modulated through electrostatic interactions, but can also be facilitated by other non-covalent interactions, including cation- $\pi$ interaction and hydrophobic interaction. In mussels, complex coacervation has been proposed as a physical mechanism for the secretion of underwater adhesives, but all characterized mussel adhesive proteins to date are positively charged polyelectrolytes [10]. Interestingly, it was found that near equal molar concentration of aromatic amino acid residues coexist with cationic residues in the characterized mussel adhesive proteins [10-12]. This inspired an interest in cation- $\pi$ interactions which give rise to strong attraction between cationic and aromatic moieties in aqueous solution $[13,14]$, and these interactions exploited to induce complex coacervation between two cationic polymers by overcoming electrostatic repulsion in water [11]. Elastin-like proteins are also able to form complex coacervation, but is thought to be largely driven by hydrophobic interaction [15]. These examples showcase that the family of complex coacervates may encompass a wide variation. What is the defining factor and balance of interaction leading to the phase separation into two aqueous fluids - a polyelectolyte-rich and a dilute phase - is still an active area of research. 


\section{Practical applications and biological relevance of complex coacervation}

The tendency of complex coacervates to engulf particles and molecular complexes is particularly notable in water. Complex coacervate has the ability to encapsulate most substances in water, which is attributed to a combination of factors, importantly the lower interfacial tension against water and attractive energy between the polyelectrolyte constituent and the surface [3, 4]. In 1953, Green and Lowell developed the first commercial example of coacervate fluids for encapsulation [16]. This property was exploited by them for developing their famous patent of what is known today as "carbonless copying paper". In their patent, ink oil was first encapsulated into the coacervate phase. This encapsulated ink oil was then stabilized by a cross-linker, and the microcapsules of ink oil utilized for generating carbonless paper (Figure 2).

The invention by Green and Lowell opened up the possibilities translating the complex coacervate to other useful applications. This is evidently seen over the past decades where interests in translating complex coacervate fluids to applications involving biological and practical relevance soared. There has also been tremendous research focused on utilizing the engulfing property of complex coacervate directed at biomedical applications [17-19]. In addition, complex coacervates are utilized in developing encapsulation technology of foods [20, 21] particles, cells [22], oils [16], flavors [23], fragrance, and even explosives [24].

Bungenberg-de Jong also proposed that complex coacervates have biological significance, given the superficial resemblance to both bacterial nucleoid and the prokaryotic cell membrane [25]. Related to this, a Russian Scientist, Opharin, proposed a theory that complex coacervation may have played a role in the origin of life [26]. Recently, progresses in research pertaining to marine organisms revealed the usefulness of the complex coacervation process: complex coacervation is considered a critical physical process in underwater adhesive formulation to facilitate or even enable the delivery, wetting, coating and infiltration of the biomolecular adhesive cocktail to the location of interest, relying on their low interfacial tension [27-30]. Water soluble polyelectrolytes-based underwater adhesives of marine organisms, including mussels, caddisflies and sandcastle worms are likely to be processed by complex coacervation [8, $27,31,32]$. The infiltration of protein-based complex coacervates to chitin is proposed to define the squid beak's mechanical gradient, where the stiffness gradient spans the entire range from the hydrated chitin of the soft beak to the dehydrated distal rostrum that displays maximum mechanical stiffness [33].

Fluid phase compartmentalization with striking resemblance to complex coacervate fluids has also been observed in eukaryotic cells [34] such as germline P granules [35], stress granules [36] and nucleoli [37], in which co-localization of proteins [38] and/or RNA has been reported [39-41] and links to transcriptional regulation and signal transduction suggested $[36,42]$. Recently, the term 'protein droplets' was coined to refer to a broad range of phenomena in which charged 
proteins, often of intrinsically disordered nature, associate with RNA species and undergo $\mu \mathrm{m}-$ scale phase separation to form droplets with highly concentrated proteins [9, 43]. On the one hand, the formation of protein droplets is believed to play a key role in intracellular spatial organization [44] as well as in the control of cell size and shape [45, 46]. On the other hand, some droplets are believed to undergo maturation and promote the formation of granules consisting of intrinsically disordered protein of pathological relevance [47]. While it appears that in many cases these are likely to be coacervate systems, the literature on this is confusing because systematic physical characterization of these complexes are lacking. In fact, most of these reports focused on the binding behavior between specific biopolymer constituents [39, 40, 48], while others refer to the growth of droplet as a first order phase transition [37] whose localization requires the distribution of protein concentration to be highly controlled [45, 49], with only few recent studies unraveling the physical properties of these protein droplets $[50,51]$ and even fewer on discussing the molecular [52] or structural [53] basis of this fascinating phenomenon. One of the few studies that present a systematic physical characterization of protein droplets is by Elbaum-Garfinkle et al in 2015 [9] that reports on the property of the intrinsically disordered protein LAF-1, already mentioned above, containing roughly equal amounts ( $\sim 12 \mathrm{~mol} \%)$ of negatively and positively charged residues. LAF-1 phase-separates into dense liquid droplets in vitro, which curiously displays decreased viscosity as droplet formation is maximized by increasing the salt concentration, but dissembles above a critical salt concentration. Interestingly, the addition of polyU-50 RNA to the LAF-1 droplets resulted in an even more dramatic, three-fold, decrease in the droplet-internal fluid viscosity, as verified by micro-rheology measurements, despite the suggested high affinity binding of polyU50 to LAF-1. Concurrently, FRAP studies reported on a $\sim 1.5$ and $\sim 2.4$-fold increase in mobility and diffusivity of both biopolymers, polyU-50 and LAF-1, within the droplets, further corroborating the finding of lowered viscosity and increased dynamicity in the protein droplets under conditions optimized for liquid-liquid phase separation and droplet formation. They further carried out single-molecule FRET studies that witness an altered interaction and increased dynamicity between LAF-1 and its RNA substrate during droplet formation (Figure 3), induced by increasing the LAF-1 concentration or optimizing the salt concentration. Their systematic and thorough studies clarify that their protein droplets consist of intrinsically disordered protein and RNA clearly belong to the complex coacervate family, as the biopolymer-rich complex coacervate phase is fluidic, while their formation is tuned by polyanion-polycation ratios or salt concentration, i.e. modulation of electrostatic interactions between the charged biopolymers. The intermolecular dynamics between LAF-1 and RNA is expected to somehow contribute to the fluidization of LAF-1, as witnessed by the systematic lowering of the droplet fluid viscosity. We speculate that this is because the stronger pairing of LAF-1 and RNA results in better charge compensation to yield lower-, but still net-charged polyelectrolyte complexes whose weak interpolymer complex interaction and weak polymer complex-water interaction contribute to a depressed cohesive energy and viscosity of the complex coacervate fluid phase, which will be subject of further discussion in this review. We note that both a decreased, as observed in the 
LAF-1 study, as well only a slightly increased fluid viscosity, as observed in other studies to be discussed [54], upon coacervate formation is noteworthy, as the coacervate is a fluid phase containing high concentrations of condensed polyelectrolytes that intuitively would be expected to yield high fluid viscosity.

Taken together, roughly three major types of complex coacervate systems have been intensively studied in the literature to date, namely (i) polyelectrolyte-polyelectrolyte (PE-PE) systems, (ii) polyelectrolyte - charged surfactant (PE-Micelle) systems [55-57], and (iii) zwitterionic molecule/polymer systems [58-62]. The droplets formed between intrinsically disordered proteins and RNA, broadly speaking, still belong to PE-PE complex coacervate systems, even though their constituents and properties may be quite different compared to synthetic polymer- or protein-polymer based PE-PE complex coacervates. We point out that this review is focused mainly on discussing the properties of PE-PE complex coacervate systems including polymer and protein constituents to maintain a manageable scope for communicating our perspectives, while we discuss selected examples of PE-micellar coacervate systems in which ultrastructures have been identified within their dense fluid phase.

\section{Physical basis and measurement of low interfacial tension of complex coacervates}

\section{4-A Low interfacial tension is a defining physical property of complex coacervates}

The examples discussed so far focuses our attention and interest to a defining property of complex coacervate fluids, their innate low interfacial tension with respect to water and wetting propensity with respect to solid surfaces $[11,30,63]$. Many applications mentioned so far rely on these properties, be it for encapsulation, phase-separation, coating and molecular transport or exchange. The interfacial tension is the energy density required to create an area with this fluid at the interface to a material or fluid of interest. It is an essential physical quantity that contributes to the engulfing property of complex coacervates. When comparing to the interfacial tension between pure liquid water and its vapor which is about $72 \mathrm{mN} / \mathrm{m}$ at $25^{\circ} \mathrm{C}$ [64], the interfacial tension of the dense complex coacervate phase with respect to the dilute equilibrium phase, its value is reduced to $10^{-3} \sim 10 \mathrm{mN} / \mathrm{m}[51,63,65-68]$. While the biphasic interfacial tension relates to the curvature of the coacervate phase droplet under pressure, the interfacial tension to other material surfaces relates to the contact angle and wetting property, which is crucial for achieving emulsification and encapsulation. While the former can be easier measured, the liquid-solid interfacial tension is difficult to access experimentally under wetting-prone condition. Thus, systematic experimental studies of the interfacial tension of the complex coacervate fluid are scarce and the understanding of the molecular origin or structural basis of the low interfacial tension of complex coacervates not mature.

\section{4-B Literature on experimental access to low interfacial tension in complex coacervate}




\section{fluids}

As complex coacervation is a phenomenon driven or regulated by electrostatic interactions between oppositely charged polymers or their complexes, it makes sense that the interfacial tension of the complex coacervate fluid was found to strongly depend on these properties, for example, the degree of polymerization (polymer chain length), linear charge density of polymer, charge distribution along the polymer chain, types of charge as well as salt concentration [69]. Weaker electrostatic attraction between weakly charged polyelectrolyte chains tends to decrease the interfacial tension. In the same vein, higher salt concentration, within the range of complex coacervating conditions, tends to reduce the interfacial tension. The interfacial tension of the complex coacervate gradually decreases by the addition of salt until it eventually diminishes entirely at above a certain critical salt concentration [67, 69-72]. Experimental data suggests the exponent of the salt concentration to the critical concentration value to be $\frac{3}{2}$, i.e. following $\gamma \sim\left(\mathrm{c}_{\mathrm{crit}}-c\right)^{3 / 2}$. However, this parameter space has by far not been thoroughly examined, so that the role of polymer flexibility and architecture, or even simpler the role of charge distribution within a polyelectrolyte chain in modulating phase separation and tuning the interfacial tension is not well understood.

A recent study of our own has set out to shed light on this fascinating complex materials and found that an exceptionally low cohesive energy of the dense complex coacervate fluid may underlie its low interfacial tension [54]. The authors of this review took an integrated approach to arrive at this conclusion, as will be discussed, namely (i) the application of state of the art and novel spectroscopic methods relying on spin labeling and electron paramagnetic resonance (EPR) to extract polyelectrolyte chain dynamics and packing, as well as local water dynamics within the complex coacervate phase [73-75], (ii) the application of state of the art characterization methods, including cryo-electron microscopy (cryo-EM) and the measurement of interfacial tension by the pendant drop method, and (iii) the rationalization with a theoretical framework suitable for a complex coacervate fluid displaying a bifluidic network structure. Crucially, the complex coacervate fluid phase was found to display bulk-like water dynamics, which at first is counter-intuitive, given the dense and concentrated nature of the complex coacervate fluid phase. This peculiar finding could be reconciled by the observation of a bifluidic network structure within the dense complex coacervate fluid phase, with a polyelectrolyte complex-rich and bulk water-like phase percolating each other at tens to hundreds of nm lengthscale separation [30, 54]. Within this network structure, water-water, polyelectrolyte complex-water and interpolyelectrolyte complex interaction was found to be weak, constituting a net low cohesive energy of the complex coacervate fluid and yielding a net low viscosity fluid [54]. The low water-water interaction was directly derived from high water diffusivity within the complex coacervate phase, the low polyelectrolyte complex-water interaction deduced from high water diffusivity found within a highly polyelectrolyte-dense phase, and low inter-polyelectrolyte complex interaction identified from unhindered polymer mobility according to EPR lineshape 
analysis of polymer-tethered spin labels $[54,75]$. The purpose of this review is to report on our perspective of how molecular-level interactions and polymer dynamics, as well as water dynamics, within the dense complex coacervate phase link to its macroscopic physical properties and how one might view the cause or effect of microphase separation and formation of fluidic ultrastructures within the complex coacervate fluid phase in this context.

\section{4-C Experimental methods of measuring interfacial tension of coacervate fluids}

A frequently encountered experimental challenge in generating a complex coacervate macrophase hinders the progress in systematically and accurately measuring the interfacial tension of the complex coacervate phase. Under complex coacervate forming condition, achieving microphase separation (Figure 1, middle panel) is straightforward compared to achieving macrophase separation (Figure 1, right panel). Challenges include the low biopolymer quantity available and/or small density differences between the dense complex coacervate and equilibrium phase. Still, given its importance, there are several studies in the literature that outline methods for measuring the interfacial tension of complex coacervate fluids, as we present here.

In the early days, Bungenberg-de Jong and his colleagues tried to measure the interfacial tension of a coacervate fluid by using Du Nouy's ring method, capillary rise method, and pendant drop method, but the results obtained with these methods were reported to be unsatisfactory given the extraordinarily small values found for the interfacial tension. At last De Ruiter and Bungenberg-de Jong succeeded to report for the first time, 70 years ago, on an ultralow interfacial tension of complex coacervate fluids $(\sim 1 \mu \mathrm{N} / \mathrm{m})$ by modifying the capillary rise method, by adapting two short capillary tubes of different radii [68]. (Figure 4A). They used the following equation to determine the interfacial tension $(\gamma)$ of the coacervate fluid:

$$
\gamma=\frac{1}{2}\left(d_{c}-d_{e}\right) g\left(h_{1}-h_{2}\right)\left(\frac{r_{1} r_{2}}{r_{1}-r_{2}}\right)
$$

In equation (1), $d_{c}$ and $d_{e}$ are the density of dense and dilute phases, as shown in Figure 1, right panel, respectively. $h$ and $r$ represent the height to which the coacervate rises in the capillary tube and the radius of capillary tube, respectively. By varying the salt concentration, buffer $\mathrm{pH}$, salt type, and ratio between gelatin (cationic polymer) and gum arabic (anionic polymer), the interfacial tension of the coacervate fluid was monitored. The highest interfacial tension was found at a mixing ratio between gelatin and gum arabic producing maximum coacervation. Interestingly, they reported that the interfacial tension decreased when increasing the salt concentration in the buffer. However, they also mentioned that their results were inaccurate due to severe variations 
given the dynamic nature of the coacervate phase and low optical contrast between water and the coacervate phase, attributed to instrumental limitations at the time.

The second method we introduce is the pendant drop method Figure 4(b)[76]. Bungenberg-de Jong and his collegues failed to use this method for the coacervate fluid at the time, but now, the pendant drop method is applicable for bulk phase separated complex coacervate fluids due to advances in image processing technology and numerical computation. The core approach is to derive the interfacial tension from the pendant drop shape of the fluid held within another fluid, typically water. When the pendant drop is in equilibrium with the surrounding fluid medium, then the vertical and horizontal force becomes balanced. Then the pressure difference (Laplace's pressure), $P$, to be accounted for comes from the boundary tension, $\gamma$, and the mean curvature $\left(\frac{1}{R}+\frac{1}{R^{\prime}}\right)$. As derived from the equations of Thomas Young and Pierre Laplace, the following equations are obtained:

$$
\begin{aligned}
& P=\gamma\left(\frac{1}{R}+\frac{1}{R^{\prime}}\right), \\
& 2 \pi x \gamma \sin \phi=V \sigma g+\pi x^{2} P,
\end{aligned}
$$

where $P$ is the pressure, $\gamma$ is the interfacial tension, $x$ is horizontal distance from the axis, $R$ and $R^{\prime}$ are the principal radii of curvature, $\phi$ is the contact angle, $V$ is velocity potential, $g$ is acceleration of gravity and $\sigma$ is density difference of the two mediums. A simplified version of this equation to find the interfacial tension has been derived by Andreas and his colleagues [76]:

$$
\gamma=\frac{g \sigma d_{e}^{2}}{H}
$$

Here, to find the boundary tension or the interfacial tension, $\gamma$, the variable to be considered is the acceleration of gravity, $g$ and the density difference of the two mediums, $\sigma$. As seen in Figure. 4(b), the maximum horizontal diameter, $d_{e}$ of the drop and the radius of the curvature can be determined from the acquired image of the pendant drop. $H$ in the equation is a function of $S$, which is the ratio of $d_{e}$ and $d_{s}$. By computing the $H$ values as the function of $d_{e}$, one can obtain a curve relating the interfacial tension as given in Equation 2(a). This method generates consistent results with the theoretical curve obtained by using the differential equation by Bashforth and Adams [77], displaying only a \pm 0.5 percent uncertainty in the determined interfacial tension value, given that the linear dimension of the droplet has a very small error of less than \pm 0.1 percent. The pendant drop method has been used by Ducel and his colleagues in measuring the interfacial tension of plant proteins ( $\alpha$-gliadin $\&$ pea globulin)-arabic gum coacervate against oil [63] (yielding $\sim 2$ to $42 \mathrm{mN} / \mathrm{m}$ ) as well as by the authors in a recent publication of a mussel adhesive protein-1 (mfp-1) and hyaluronic acid (HA) coacervate against water (yielding $\sim 0.2$ to $1.4 \mathrm{mN} / \mathrm{m}$ ) [54]. However, when the interfacial tension of the coacervate fluid is too low (typically $<0.1 \mathrm{mN} / \mathrm{m}$ ) to sustain a stable hanging drop in the equilibrium fluid phase, the pendant drop method fails. 
Recently, researchers have also reported on precise measurements of interfacial tension of the complex coacervate fluid phase relying on nanomechanics, owing to technical advancement in this area. The first nanomechanical method is colloidal probe atomic force microscopy (CP-AFM) [78, 79]. CP-AFM method is based on the principle of capillary condensation. In a system that is nearly saturated with one component, a capillary bridge consisting of a condensed phase of this component can form spontaneously between two surfaces if the condensed phase wets the surfaces, as shown schematically in Figure 4(c). This phenomenon causes, for example, the adhesion between sand grains in humid air, and makes the sand stick together so it can be shaped. If the component is a non-wetting phase for the surface, the phenomenon of capillary evaporation, also known as cavitation, can take place. The first study of using CP-AFM in measuring interfacial tension of an aqueous solution was carried out by Sprakel and his colleagues [80]. The mathematical description for interfacial tension as measured by CP-AFM of the coacervate phase of poly(2-(methacryloyloxy)-ethyl-trimethylammonium chloride) (PMETAC) and poly(3-sulfopropyl methacrylate) (PSPM) has been presented by Spruijt et al. [67]. Briefly, there are generally two contributions to the capillary force: the Laplace pressure difference across the interface and the interfacial tension acting to minimize the interfacial area.

$$
F=\pi r_{1}^{2} \gamma\left(\frac{1}{r_{1}}-\frac{1}{r_{2}}\right)-2 \pi r_{1} \gamma \cos \theta
$$

where $F$ is the capillary force, $\gamma$ is the interfacial tension, $\theta$ is the contact angle at the two phase contact line between the coacervate and the solid probe in water and $r_{1}$ and $r_{2}$ are the principal radii of curvature, as shown in Figure 4(c). They reported the interfacial tension of the coacervate phase to be of order $0.1 \mathrm{mN} / \mathrm{m}$, while finding a sharp drop in the interfacial tension to vanish at a critical value, as salt concentrations increases in the coacervate system. In such study of a solid (probe)-liquid interface, the precise measurement of the contact angle is critical for the precise measurement of the interfacial tension. This is achieved by placing coacervate microdroplets dispersed in equilibrium phase (Figure 1, middle panel) in a liquid cell with the target flat surface placed at the bottom, where the contact angles of sedimented coacervate droplets on the surface is measured over a certain period of the time with a contact goniometer. Another example of employing CP-AFM for measuring interfacial energies can be found in the study of Cha and colleagues of a complex coacervate fluid made of mfp-151 and HA [51]. In their study the interfacial tension of the coacervate phase was found to be low as well $(\lesssim 0.3 \mathrm{mN} / \mathrm{m})$, and to follow the Hofmeister ordering for ion effects [81].

The last method to be discussed here is the use of the surface forces apparatus (SFA). SFA has been used for many years to measure the physical forces between various materials and molecular systems, including the lateral and normal forces in and of complex fluids at the interface to mica, self- assembled monolayers, lipid bilayers and other surfaces [82]. SFA has been used to monitor the physical properties of the coacervate phase, while requiring very small 
volumes ( $\sim 50 \mu \mathrm{L}$ liquid) [30]. SFA has also been used to gain insight into the wetting and spreading of the coacervate fluid phase on the contacting solid surface and to accurately measure forces as low as $10 \mathrm{nN}$, as a function of surface separations with Angstrom resolution by using multiple beam interference fringes of equal chromatic order (FECO). The use of SFA is illustrated in Figure 4 (d), where the dense coacervate phase is formed and confined between two mica surfaces. The thickness of the confined coacervate phase or the gap distance between the two mica surfaces can be monitored in real time using the FECO fringes. The two surfaces are brought together to permit the coacervate fluid film to coalesce and make contact with the opposing mica surface and to spread. As the coacervate spreads on the second mica surface, it forms a continuous viscous bridge as shown in Figure 4(d). The FECO fringes and microscopic top-views are continuously video-recorded during the experiment. For reference, FECO patterns should be smooth and continuous when the liquid phase trapped in the gap between two mica surfaces is homogeneous, whereas the FECO patterns displays discontinuities when the aqueous phase is heterogeneous due to changes in the local refractive index and/or film roughness. The capillary adhesion associated with a coacervate meniscus bridge between two mica surfaces was measured upon separation of the two surfaces coated with the coacervate fluid. The determination of this adhesion force enabled the determination of the surface tension value $\gamma$ at the coacervate phase-water interface using the following equation: $\gamma=\frac{F_{a d} \cos \theta}{4 \pi R}$, where $F_{a d}$ is the adhesive force measured by SFA during separation and $R$ the curvature of the disk that mica has been glued to, while the assumption $\cos \theta \simeq 1$ is assumed valid in the systems studied, as complete wetting of the mica surface with the coacervate fluid phase is ensured, facilitated by both favorable polyelectrolyte-surface interaction and sufficiently low surface tension of the fluid to be studied. This method has been used by Hwang, Zeng, Waite, Israelachvili, Tirrell and coworkers $[30,50,83]$ to measure the interfacial tension of the coacervate phase constituting recombinant mussel adhesive proteins and HA. Interfacial energies of the coacervate fluid was found low $(0.3 \sim 1.0 \mathrm{mN} / \mathrm{m})$. Relying on similar SFA measurements, Priftis and colleagues [50] have also revealed the dependence of the interfacial tension on salt concentrations and the polyelectrolyte chain length in complex coacervate system composed of Poly(l-lysine hydrochloride) (PLL) and poly(l-glutamic acid) (PGA). The interfacial tensions of these coacervate fluids generally decrease as salt concentration increases, or as the chain length decreases, i.e. upon weakening the inter-polyelectrolyte electrostatic interaction. Specifically, they found that the interfacial tension of the coacervate phase fluid decreases from $0.98 \mathrm{mN} /$ $\mathrm{m}$ to $0.35 \mathrm{mN} / \mathrm{m}$ upon increasing the $\mathrm{NaCl}$ salt concentration from $100 \mathrm{mM}$ to $600 \mathrm{mM}$, and consistently increases from $0.7,1.0$ to $4.4 \mathrm{mN} / \mathrm{m}$ upon increasing the degree of polymerization from 30,200 , to 400 .

\section{4-C Theoretical consideration of the interfacial tension of coacervate fluids}

The theoretical treatment and analysis are based on equilibrium thermodyamics and statistics, 
except when taking a molecular dynamics simulation approach, the latter of which is only applicable to study the complexation of small systems. The most common approach to treat complex coacervation is based on the Voorn-Overbeek theory [84] which combines the FloryHuggins entropy of mixing and the electrostatic self-energy at the Debye-Huckel (DH) level to yield an expression for the Flory-Huggins free energy, $F$, following,

$$
\frac{F}{N k_{B} T}=\sum_{i} \frac{\phi_{i}}{r_{i}} \log \left(\phi_{i}\right)+\alpha\left(\sum_{i} \sigma_{i} \phi_{i}\right)^{3 / 2} .
$$

Here, $r_{i}$ is the number of lattice elements occupied by the $\mathrm{i}^{\text {th }}$ component which can be polyelectrolyte, salt or solvent components. $\phi_{i}$ is the volume fraction of the $i^{\text {th }}$ component, and $N$ is the ratio of the total system volume $(V)$ and solvent volume $(v)$, ie. $N=\frac{V}{v}$. Further, $\alpha$ describes an interaction strength defined as $\frac{16 \pi^{2}}{3}\left(\frac{l_{B}^{3}}{v}\right)^{\frac{1}{2}}$, where $l_{B}=\frac{e^{2}}{4 \pi \epsilon_{0} \epsilon_{r} k_{B} T}$ is the Bjerrum length and $\sigma_{i}$ the average charge density of the $\mathrm{i}^{\text {th }}$ component referred to above. The first term represents the mixing entropy of the polymer and water components, while the second term comes from the electrostatic self-energy within the DH approximation. Qin and colleagues [70] applied this approach and calculated the interfacial tension assuming the Cahn-Hilliard theory for the interfacial profile perpendicular direction to the interface $z$ [85],

$$
\gamma=\frac{k_{B} T}{v} \int_{-\infty}^{+\infty} d z\left(f_{0}[\phi(z)]-f_{0}\left[\phi_{0}\right]+\frac{1}{2} B\left(\frac{d \phi}{d z}\right)^{2}\right)
$$

Here, $f_{0}$ is the local part of the free energy and corresponds to the bulk polymer complex concentration. $B$ is related to the thickness of the interface of the coacervate macrophase boundary which is equivalent to $\frac{\sigma_{0}^{2}}{18 \phi}$, where $\sigma_{0}$ is the segmental length of the polymer, $\phi$ is a volume fraction of polymer complex. Despite the fact that this theoretical treatment greatly simplifies the polyelectrolyte complex and solution system, they could successfully explain the power exponent of the dependency of the interfacial tension on salt concentration following, $\gamma=$ $\gamma_{0}\left(1-\frac{c}{c_{c r i t}}\right)^{\frac{3}{2}}$. The meaning of the two fitting parameters $\gamma_{0}$ and $c_{c r i t}$ are interfacial tension at zero salt concentration and the critical salt concentration, respectively. Although this model is conceptually intuitive, it has drawbacks that it contains phenomenological fitting parameters.

Efforts to go beyond this approximation are still challenging. Borue and Erukhimovich [86] proposed that symmetric polyelectrolyte complexes exist in the form of globules due to the interplay of excluded volume interaction and the fluctuation-induced Coulomb interaction, where the complex coacervate may be an aggregate of these globular polyelectrolyte complexes. Their theory considers the fluctuation of Coulombic energy within a random phase approximation (RPA) level, which includes the fluctuation of field up to second order, i.e. includes Gaussian fluctuations. The interfacial profile of these globule is obtained from Lifshitz-Grosberg theory [87]. 
Simulation is a powerful way to include higher order fluctuations of fields and the degrees of freedom beyond the RPA level approximation. The basic idea of a simulation is to obtain the thermodynamical quantities by the integration of partition functions, numerically. The most common sampling strategies are Monte Carlo and molecular dynamics simulations. However, these particle-based simulations are limited by small spatial and temporal scales, and thus are not successful to observe the macroscopic phase separation of complex coacervate. Field theoretic simulation (FTS) is a good alternative to overcome these problems [88]. The particle representation of partition function is translated into a field-based form after a HubbardStratonovich transformation [89]. The mean field solution is obtained by replacing the Hamiltonian with saddle point values. In order to include higher order fluctuations, Riggleman, Kumar and Fredrickson [69] sampled the field configurations by using a complex Langevin method that transforms the integration of the partition function to a stochastic differential equation in Langevin dynamics. Going beyond the mean field treatment of field theory for highly charged systems can cause UV divergence. To avoid the UV divergence issue, distributed charges are considered instead of point-like charges [90]. Both, the mechanical interfacial tension and the free energy change with respect to the areal change are measured. They show that FTS can explain the scaling of the effective Bjerrum length and salt concentration with respect to interfacial tension. Although this approach is powerful, it requires very fine grids to consider sufficiently high resolution of the charge configuration. Furthermore, strong coupling effects of electrostatic interactions may not be fully considered.

Despite the progress made, the approaches discussed thus far miss important physics of complex coacervate fluids, such as ion hydration [91], strong coupling effects [92] and charge renormalization $[93,94]$. It is unlikely to obtain self-consistent approaches when considering these effects, due to the hierarchical physics spanning multiple spatial and temporal scales. Some effort to combine part of these properties are still on going. For example, the liquid state theory of polymer is introduced to capture the charge release [95] or the strong coupling effect [96].

In light of the author's recent experimental observation of nanometer scale bifluidic phase separation within the dense complex coacervate phase, as witnessed by high-pressure cryogenic transmission electron microscopy (cryo-TEM, Figure 5) and substantiated by the measurement of bulk-like water dynamics to be discussed next [54], it became clear that in some systems the dense complex coacervate phase itself may not be a homogeneous phase, but is phase-separating again into a a polyelectrolyte complex-rich and a bulk-like water microphase at tens to hundreds of nm length scales for phase separation. Thus, there was a need to do the first step towards a theoretical framework that can rationalize the interactions underlying such a dynamic network system, with the water and polyelectroeelyte complex-phase percolating within a bifluidic system. The experimental observations provide a rationale for certain assumptions made in the following model, namely (i) that the polycation and polyanion form correlated complexes, and thus will be considered together as a polyelectrolyte complex component, (ii) that the interpolyelectrolyte complex interaction is of electrostatic nature, but weakened due to significant 
(but still incomplete) charge compensation, and (iii) that the polyelectrolyte complex-water interaction is weak, as well as the water-water interaction is bulk water-like, i.e. essentially indistinguishable to that of the equilibrium water phase [54]. The goal of this study was to compare the predicted and experimental physical properties of such a complex fluid system, most importantly the dependency of the interfacial tension of the polyelectrolyte-rich fluid phase on water content, polyelectrolyte complex-water and inter-polyelectrolyte complex interaction strength to learn about the driving forces at the molecular level that may lead to the intriguing observation of a bifluidic structure within the dense complex coacervate fluid. We took a simplistic approach to consider both the polymer and water phase within the dense complex coacervate phase by integrating out any spatial variation, i.e. considering the polymer and water phase as invariant over the volume of interest similarly done in Spruijt et. al.[97]. Under this assumption, we may include this effect with the following additional term $\chi_{p w} \phi_{p} \phi_{w}$ in the Flory-Huggins expression for free energy, F, shown earlier (Eq. 5), where $\chi_{p w}$ is the polyelectrolyte complex-water interaction parameter, $\phi_{p}$ the polymer volume fraction $\phi_{w}$ the water volume fraction. By taking the interfacial profile model from Cahn and Hilliard theory (Eq. 6 ), we obtain the dependence of the interfacial tension, $\gamma$, on the interaction strength between the

polyelectrolyte complex and water, $\chi_{p w}$, following $\gamma=\gamma_{0}\left(1-\frac{3}{2} \chi_{p w}\right)$. Here $\chi_{p w}$ is a negative value for attractive interaction, so that weak repulsive interaction leads to small decrease of the $\gamma$ values, weak attraction to small positive increases of the $\gamma$ values, while strong attraction leads to larger positive increases to $\gamma$. This model thus provides a sensible rationale that weaker interaction between the polyelectrolyte complexes and water can result in smaller interfacial tension. Notably, the interfacial tension of complex coacervate fluid under optimal coacervating conditions is typically significantly lower because it reduces the difference between two phases. It is typically achieved with lowering salt concentration or a mismatch in polycation and polyanion constituents, both leading to increases in $\chi_{p w}$. It is interesting to notice that $\chi_{p w}$ can affect the critical salt concentration [97].

\section{Complex coacervate with a bicontinuous fluid structure and ultra-low cohesive energy}

Despite significant interest in and importance of the low interfacial tension of complex coacervate fluids, as highlighted, few systematic experimental studies exist that report on its physical basis, and even fewer that comprehensively combine experiments and theoretical considerations [54, 74, 98-100]. We will highlight one most recent example published by the authors that showcase how one can gain access to key physical properties of complex coacervate fluids. The key approach is to rely on innovative electron paramagnetic resonance (EPR)-based spectroscopic measurements to extract solvent and polymer packing dynamics by utilizing free or polymer-tethered spin probes based on stable nitroxide radicals [74, 75]. These studies are combined with systematic measurements of interfacial tension of the complex coacervate fluid 
using the pendant drop method, high-pressure cryo-TEM of the complex coacervate fluid phase and a theoretical model for treating the complex coacervate fluid phase [54]. In this study, complex coacervates are formed from mfp-1 and HA in the presence of $700 \mathrm{mM}$ salt and a 45:55 mfp-1:HA ratio. Mfp-1 is a cationic mussel adhesive protein with extended polyproline type II helical structure in water [101-103]. What distinguishes this study from previous spectroscopic investigation of complex coacervate fluids by Han and coworkers $[39,74,104]$ is that the nature of this complex coacervate fluid and the expression yield of mfp-1 permitted the successful macroscopic phase separation of the complex coacervate into a dense complex coacervate and a dilute equilibrium phase (Figure 6(a)), granting their separate and detailed characterization. This includes the use of freely dissolved spin probes in the respective equilibrium phases for their separate examination of average fluid property as seen by a small molecule probe. In addition, spin probes covalently tethered to mfp-1 [54, 75], as well as HA [75] have been employed for the study of complex coacervate fluids, before and after macroscopic phase separation.

For the characterization of these fluids, EPR lineshape analysis was used to extract polymer packing dynamics using polymer-tethered spin labels and fluid dynamics using free spin probes. The exceptional sensitivity of spin label dynamics in the tens of ps to nanoseconds timescale for tumbling has been widely $[105,106]$ used to quantify spin label tumbling rate, and extract from there either polymer backbone dynamics if the spin label is rigidly bound or tethered with limiting degrees of freedom to the polymer chain, or polymer packing dynamics of collapsing or assembling systems, even if the spin label is tethered with significant rotational freedom. Information of the latter type can also be obtained using free spin labels, depending on the question at hand - for example, the free spin probe dynamics would sensitively report on viscous or confined environments, while the spin label tethered to the polymer chain would provide more local information on the packing of the polymer chain itself [54, 107-110].

Concurrent to spin label dynamics-derived polymer dynamics, Overhauser dynamic nuclear polarization (ODNP)-enhanced ${ }^{1} \mathrm{H}$ nuclear magnetic resonance (NMR) relaxometry was used to extract local water dynamics near the polymer-tethered spin labels, as well as near free spin probes selectively partitioned into the macrophase-separated complex coacervate fluid $[54,75]$. ${ }^{1} \mathrm{H}$ ODNP of water at the EPR Larmor frequency of $\sim 10 \mathrm{GHz}$ measures the dipolar coupling factor between the spin probe and water that is sensitive to diffusion motion of water within 3-10 $\AA$ from the spin probe, in the tens to hundreds of ps timescale-encompassing faster than bulk water diffusivity up to 100 -fold retardation $[73,111]$. In the specific study discussed here, the macrophase-separation of the complex coacervate consisting of mpf- 1 and HA biopolymers permitted the use of free spin probes, such as 4-hydroxy (4OH) TEMPO, or spin labeled polyethylene glycol (PEG) molecules with different molecular weights, to obtain solvent fluid dynamics in the two separated phases as seen by these probes [54].

Furthermore, the access to macrophase-separated complex coacervate fluids allowed us to easily determine the total polyelectrolyte densities in each phase, which was found to be 1000 
$\mathrm{mg} / \mathrm{mL}$ in the macrophase-separated dense complex coacervate fluid, i.e. 4 orders of magnitude higher than that in the dilute equilibrium phase $(\sim 0.1 \mathrm{mg} / \mathrm{mL})$, according to measurements relying on an amino acid analyzer. Thus, it was the more surprising to find that EPR and ODNP measurements using the free spin probe $4 \mathrm{OH}$ TEMPO revealed that the water diffusivity measured by its dipolar coupling efficiency to spin probes with an EPR Larmor frequency at $\sim 10$ $\mathrm{GHz}$ and the effective fluid viscosity as evaluated by the rotational degree of freedom of $4 \mathrm{OH}$ TEMPO inside the dense complex coacervate phase was indistinguishable from that in the dilute supernatant phase (Figure 6b).

Because of this peculiarity and what it implies, we discuss in more detail the observation of bulk-like water dynamics within the polyelectrolyte-rich fluid phase of $1000 \mathrm{mg}$ polyelectrolytes per $\mathrm{mL}$ fluid concentration - an unexpected observation. In fact, water within the solution of any other biopolymer-rich fluid at this concentration would essentially constitute hydration water, namely water interacting within $1 \mathrm{~nm}$ with the polymer surface, resulting in retarded hydration water diffusion dynamics due to strong biopolymer-water attraction $[112,113]$. However, what we repeatedly observe is high local water content, high local water diffusivity, as well as directly or indirectly high polymer degrees of freedom within the complex coacervate phase across various systems, which is counter to an intuitively expected property of dehydrated polymer fluids with high fluid viscosity and slowed water diffusivity [74, 75, 104]. While we have been speculating about the existence of "water puddles" within the dense complex coacervate fluid phase previously, the limitation of determining complex coacervate properties within microphase-separated fluids using polymer-tethered spin labels left doubts whether the measurements might be skewed from effects of exchange between the dense coacervate and the essentially bulk water equilibrium phase. Thus, the access to the macrophase-separated, polyelectrolyte-rich, fluid phase of $1000 \mathrm{mg} / \mathrm{ml}$ offered a powerful opportunity to verify past measurements of water and polyelectrolyte dynamics made of microphase-separated complex coacervate fluids consisting of mfp-1 and HA biopolymers [75] that relied on the use of covalently tethered spin labels to the mfp-1 or HA biopolymer to access local information from the complex coacervate fluid. Gratifyingly, we find comparable results with spin labeled mfp1within microphase-separated complex coacervate fluids as reported on in previous studies [75], namely only a small (in this case 2-fold) diffusion retardation of water observed near the mfp-1 protein surface (within $1 \mathrm{~nm}$ ) in the dense complex coacervate phase compared to the dilute equilibrium phase [54]. Cw EPR lineshape shows that the spin label tethered to the mfp-1 surface remains mobile in the dense complex coacervate phase, with features representative of fully solvent-exposed protein or polymer surfaces. Thus, the mfp-1 protein remains fully hydrated and non-aggregated in solution state, even as it forms a highly dense polyelectrolyte complex phase, and further phase-separates from water within the dense complex coacervate phase.

The observation of the presence of water dynamics indistinguishable from that of bulk within the densely crowded complex coacervate phase $(1000 \mathrm{mg} / \mathrm{ml}$ polyelectrolyte concentration $)$ can only be reconciled, to the author's mind, with the presence of a heterogeneous ultrastructure 
within the complex coacervate phase. Experimentally, there are scattering (SANS mainly) studies in the literature reporting on the mesh size / correlation length of agar-gelatin complex coacervate to be $1.2 \mathrm{~nm}$ [114] and of PDADMAC-BSA coacervates to be 7.5 8.1 nm [55]. These same studies report on the size of porous heterogeneity of the complex coacervate to be of $\sim 20 \mathrm{~nm}$ [114] and 100-1000 nm [55] lengthscales. There are other cryo EM methods reporting on porous structures of complex coacervates at 100-200 nm lengthscales [59]. In other words, there clearly is a distribution of lengthscales. High pressure cryo-TEM combined with differential staining verified this hypothesis, not only by displaying a heterogeneous nanophaseseparated (tens to $100 \mathrm{~nm}$ lengthescale) and interconnected bicontinuous network, but also by validating that one phase is water and the other the polyelectrolyte-rich phase (Figure 6(c-d)).

While qualitative image analysis suggests that the water and polyelectrolyte-rich phase may occupy similar fluid volumes, a more direct and quantitative analysis was carried out by signal integration of EPR of the 4OH TEMPO probe that predominantly partitions into the aqueous phase to extract polyelectrolyte- and water volume fractions. Double integration of the (derivative) EPR spectra finds that the water content inside the dense complex coacervate phase over that in the (effectively bulk water) equilibrium phase is about $50 \%(\mathrm{v} / \mathrm{v})$. Thus, we conclude that the ultrastructure of the mfp-1/HA complex coacervate phase resembles a water-filled sponge like network structure.

To test the property of such a bifluidic water-filled sponge structure, three different sizes (and shape) of spin labeled PEG polymers were added as copolymers to the macrophaseseparated complex coacervate phase. PEG was employed as it is a classic additive to exert excluded volume effects by dehydrating its aqueous environment and/or as viscogens as it binds and retards solvent diffusivity. ODNP results validated that water retardation near the surface of these spin-labeled PEG probes in water increases with PEG size and bulkiness (Figure 6b), consistent with assumptions in the literature on strong PEG-water interaction [115]. When added to the macrophase-separated complex coacervate phase, ODNP finds stronger retardation of PEG-surface water diffusivity compared to in the equilibrium phase (Figure 6b), while interestingly, cw EPR lineshape reports on spin probe rotational freedom indistinguishable from that in the dilute equilibrium phase. In other words, the spin label on the PEG surface somehow experiences an altered, slower, water solvent property in the dense complex coacervate while it does not strongly interact with the polyelectrolyte framework. The fraction of spin labeled PEG, as quantified by the double integration of EPR spectra, shows that an increasingly smaller fraction of PEG with increasing size or bulkiness partitiones into the complex coacervate fluid phase. This result would be consistent with mesh size-selective partitioning of PEG within the complex coacervate spong structure. However surprisingly, the cryo-TEM results clearly show that even small concentrations of PEG added consistently and repeatedly abolish the bicontinuous fluid structure entirely (Figure 6(c-e)). Considering there was no interaction observed between PEG and the polyelectrolyte network (not surprisingly given the strong hydration layer of PEG), what is remained to cause the destruction of the ultrastructure is 
enhanced water-water cohesiveness caused by adding PEG (consistent with its role as a viscogen or crowding agent) that disturbs the delicate balance of water-water and water-polyelectrolyte complex interactions constituting the low cohesive energy of this complex fluid.

Crucially, the abolishing of the ultrastructure in the presence of $20 \mathrm{kDa}$ PEG was concurrently observed with a significant increase in the interfacial tension of the comple coacervate fluid relateive to water from $\sim 0.2 \mathrm{mN} / \mathrm{m}$ in the absence of PEG to $\sim 1.5 \mathrm{mN} / \mathrm{m}$ in the presence of $20 \mathrm{kDa}$ PEG, as measured by the pendant drop method (Figure 6(c-e)) [54]. We conclude that the abolishing of the bifluidic and bicontinuous ultra structure and the increase in interfacial tension in the presence of PEG are closely linked, with the strengthening of the cohesiveness of water achieved by the addition of the viscogen, PEG. In other words, generating fluids with low cohesiveness and a bifluidic and bicontinuous network phase may be an essential design consideration for achieving ultra-low interfacial energy in complex coacervate fluids.

Taken together, weak water-water, polyelectrolyte complex-water and inter-polyelectrolyte complex interaction constitute a net low cohesive energy for the complex coacervate fluid, which in turn underlies the net low viscosity, high dynamicity and low interfacial tension of the complex coacervate fluid. We further suggest that a bifluidic and bicontinuous ultrastructure within the dense complex coacervate fluid offers a structural basis for achieving this peculiar observation of a surprisingly low cohesive energy within the dense complex coacervate fluid phase containing $1000 \mathrm{mg}$ polyelectrolytes per $1 \mathrm{~mL}$ fluid (density $\sim 1.8 \mathrm{~g} / \mathrm{mL}$ ). In fact, the suggestion that the complex coacervate fluid itself displays low cohesive energies is consistent with the observation that the complex coacervate fluid displays low interfacial tension with respect to water and typically highly favorable wetting property to surfaces in an aqueous environment, the ladder aided by the generally attractive energy between the polyelectrolyte constituents and most surfaces studied to date. Then, why is the polyelectrolyte complex-water and inter-polyelectrolyte complex interaction so weak of highly charged polyelectrolyte constituents? As the complexation and coacervation of two oppositely charged polyelectrolytes occur at relatively high ionic strength, the screening effects of added salt and the electrostatic complexation of the oppositely charged polymer moieties significantly shields residual excess charges, and thus exposes extended and effectively low-charge polyelectrolyte complexes to water. Crucially, we suggest that the coacervate phase is still held together by electrostatic interactions between polycations and polyanions, as well as even weaker electrostatic interaction between polyelectrolyte complexes, while the network interaction between surface water and the polyelectrolyte complexes is also expected to be weak.

\section{Observation of ultra-structure in coacervate fluids in the literature}

Given the surprising findings reported by the authors [54], we wish to finish with a brief review of reports on ultrastructures observed within coacervate fluids. Since complex coacervate phases are highly dynamic in water, there are only few reports on the ultra-structure of coacervate fluids. However, recent advances in the cryo-EM [11, 30, 52, 54, 59, 116-118] and neutron scattering 
$[52,104,114,119,120]$ technology have enabled the visualization of fluid structures of complex coacervates in the water phase from the early 2000s. We propose that the nature of these fluid structures is likely linked to the low interfacial property of these complex coacervate fluids. However, the validity or generality of this concept will require many more and systematic studies.

Menger and his colleagues visualized for the first time (to the best of our knowledge) a sponge-like fluid phase structure (Figure 5(a)) of complex coacervates made of a simple zwitterionic gemini surfactant (micelle system) by cryogenic scanning electron microscopy (cryo-SEM) [59]. Since then, cryo-TEM and freeze-fracture transmission electron microscopy (FF-TEM) techniques were used to observe interconnected corona-shaped structures in PEMicelle based coacervate systems $[55,116,118]$. A cryo-TEM study that reported on the protein-polyelectrolyte coacervate confirms the presence of dense, interconnected, domains at length scales of hundreds of nanometers to micrometers [52].

In PE-PE complex coacervate system, Hwang and coworkers have observed that the complex coacervate phases made of mfp- 1 and HA, or mfp- 1 and pMETAC display a bicontinuous phase structure as verified through the use of high-presssure cryo-TEM (Figure 5(b)) [11, 30, 54]. Our recent studies $[11,54]$ of different complex coacervate systems further confirm the prevalency of a bicontinuous fluid structure in complex coacervate phases as verified by cryo-TEM (Figure 5(c)). Other small angle neutron scattering (SANS) studies of complex coacervate systems, including both PE-PE and PE-ME systems, confirm the existence of a heterogeneous interconnected network structure within the complex coacervate phase $[52,104,114,119,120]$.

The past two decades have also witnessed a continuous effort on the characterization of complex coacervate ultrastructures with the help of advanced scattering methods [52, 104, 114 , 119-122]. There are small angle neutron scattering (SANS) studies of the complex coacervation of poly(acrylic acid) and poly(N,N-dimethylaminoethyl methacrylate) polymers, whose study suggests that polyelectrolytes in the dense phase adopt a conformation that closely fits the model of an ideal Gaussian chain (Figure 7) [98]. Notably, the excessive charges are likely largely screened, in this, as well as in $\beta$-lactoglobulin/pectin complex coacervates [119]. SANS also reports on the extent of strong overlapping polycation and polyanion chains to form a weakly interconnected and heterogeneous network, as discussed in systems made of agar-gelatin and a whey protein-gum arabic to form complex coacervates [123, 124]. The mesh size of the network is much smaller than the polyelectrolyte monomer's radius of gyration, and reduces as salt concentration increases, as found in a study of bovine serum albumin (BSA) and sodium polystyrene sulfonate (NaPSS) based complex coacervates studied by SANS [122]. At high salt concentration, this system resembles that of a polymer in semi-diluted solution, hinting at a fully hydrated polyelectrolyte framework. Notably, in the mentioned study of BSA and NaPSS 
complex coacervates, an ultrastructure within the complex coacervate phase forms irrespective of ionic strength or protein-polyelectrolyte ratio, indicating that this is an innate property of these complex coacervates. In this highlighted report, small angle X-rays scattering (SAXS) and small angle light scattering (SALS) methods were also applied to report on domains of varying densities inside the complex coacervate phase that were found to be much larger than the correlation length of polyelectrolyte monomers (Figure 7), revealing a sub- and micro-meter scale bicontinuous and biphasic ultrastructure. This scattering study finds that the complex coacervate phase adopts two modes of relaxations: a fast mode corresponding to the fluctuation of the mesh size and a slow mode corresponding to the diffusion of the domains of varying density. The scope of the knowledge of complex coacervate ultrastructure has been broadened by the application of advanced scattering methods, where a bifluidic and bicontinuous network ultrastructure appears to be a quite common feature found in several different complex coacervate systems.

\section{Conclusion}

The extremely low interfacial energy of complex coacervate fluids against water is its key property that dominates or defines its biological significances, as well as practical applications. In this review, we provide our perspective on the molecular and structural bases of the innate low interfacial tension of complex coacervate through the reviews of its molecular-level, physical, properties.

When the bulk phase is separated into a dilute phase and a dense coacervate phase at a complete complex coacervation stage, likely an equilibrium state, the polyelectrolyte density difference between the two phases can reach up to 10,000 times. The surprising characteristics is that the water diffusivity in these two phases is almost indistinguishable across several complex coacervate systems studied by the authors. We propose to reconcile this peculiar feature with the occurrence of a sponge-like bifluidic and bicontinuous fluid structure with $10 \mathrm{~s}$ to $100 \mathrm{~nm}$ scale phase separations, revealed by recent cryo-TEM and SANS observations of the dense coacervate phase, where one is a water-rich nanophase and the other a polyelectrolyte-rich nanophase. An integrated approach of applying a wide range of experimental studies led us conclude that the water-rich nanophase is mainly composed of unbound, bulk-like water. The characteristic property of the dense complex coacervate fluid seems to be weak interactions between waterwater, polyelectrolyte complex-water, and polyelectrolyte complex-polyelectrolyte complex, while these weak interactions are responsible for the exceptionally low interfacial energy of the complex coacervate against water and many other surfaces in aqueous environments.

Although, our current study is mainly focused on PE-PE coacervate system, it offers a valuable design consideration for fluid material with low interfacial tension in water and on wet surfaces that has the potential to address the grand challenge of wet adhesion and molecule encapsulation. 


\section{Acknowledgement}

This material is based upon work supported by the National Research Foundation of Korea (NRF-C1ABA001-2011-0029960, NRF- 2014R1A6A1031189 supporting, D.S.H, H.Y.Y, and, NRF Global PH.D Fellowship Program, NRF-2011-0008261 supporting H.Y.Y.) This research was supported by the U.S. National Science Foundation (NSF) through the MRSEC Program DMR-1121053 (MRL-UCSB). S.H. also would like to acknowledge support by the Cluster of Excellence RESOLV (EXC 1069) funded by the Deutsche Forschungsgemeinschaft. 


\section{References}

[1] Tiebackx F. Gleichzeitige ausflockung zweier kolloide. Colloid \& Polymer Science. 1911;8:198-201.

[2] Bungenberg de Jong H. Crystallisation-coacervation-flocculation. Colloid science. 1949;2:232-58.

[3] De Gennes P-G. Wetting: statics and dynamics. Reviews of modern physics. 1985;57:827.

[4] Marchand A, Weijs JH, Snoeijer JH, Andreotti B. Why is surface tension a force parallel to the interface? American Journal of Physics. 2011;79:999-1008.

[5] Abdilla A, Shi S, Burke NA, Stöver HD. Multistimuli responsive ternary polyampholytes: Formation and crosslinking of coacervates. Journal of Polymer Science Part A: Polymer Chemistry. 2016;54:2109-18.

[6] Lazko J, Popineau Y, Legrand J. Soy glycinin microcapsules by simple coacervation method. Colloids and Surfaces B: Biointerfaces. 2004;37:1-8.

[7] Gu X-L, Zhu X, Kong X-Z, Tan Y. Comparisons of simple and complex coacervations for preparation of sprayable insect sex pheromone microcapsules and release control of the encapsulated pheromone molecule. Journal of microencapsulation. 2010;27:355-64.

[8] Wei W, Tan Y, Rodriguez NRM, Yu J, Israelachvili JN, Waite JH. A mussel-derived one component adhesive coacervate. Acta biomaterialia. 2014;10:1663-70.

[9] Elbaum-Garfinkle S, Kim Y, Szczepaniak K, Chen CC-H, Eckmann CR, Myong S, et al. The disordered P granule protein LAF-1 drives phase separation into droplets with tunable viscosity and dynamics. Proceedings of the National Academy of Sciences. 2015;112:7189-94.

[10] Lee BP, Messersmith PB, Israelachvili JN, Waite JH. Mussel-inspired adhesives and coatings. Annual review of materials research. 2011;41:99.

[11] Kim S, Huang J, Lee Y, Dutta S, Yoo HY, Jung YM, et al. Complexation and coacervation of like-charged polyelectrolytes inspired by mussels. Proceedings of the National Academy of Sciences. 2016;113:E847-E53.

[12] Kim S, Faghihnejad A, Lee Y, Jho Y, Zeng H, Hwang DS. Cation- $\pi$ interaction in DOPA-deficient mussel adhesive protein mfp-1. Journal of Materials Chemistry B. 2015;3:738-43.

[13] Dougherty DA. Cation-pi interactions in chemistry and biology: a new view of benzene, Phe, Tyr, and Trp. Science. 1996;271:163.

[14] Lu Q, Oh DX, Lee Y, Jho Y, Hwang DS, Zeng H. Nanomechanics of cation- $\pi$ interactions in aqueous solution. Angewandte Chemie International Edition. 2013;52:3944-8.

[15] Muiznieks LD, Cirulis JT, van der Horst A, Reinhardt DP, Wuite GJ, Pomès R, et al. Modulated growth, stability and interactions of liquid-like coacervate assemblies of elastin. Matrix Biology. 2014;36:39-50.

[16] Green BK, Lowell S. Oil-containing microscopic capsules and method of making them. Google Patents; 1957.

[17] Stewart RJ, Kaur S, Mann L, Papanna R, Moise KJ. Crosslinkable complex coacervates for tissue repair after in utero surgery. Abstracts of papers of the American Chemical Society. Vol. 248: American Chemical Socieity 1155 16th ST, NW, Washington, DC 20036 USA; 2014.

[18] Stewart RJ, Wang CS, Shao H. Complex coacervates as a foundation for synthetic underwater adhesives. Advances in colloid and interface science. 2011;167:85-93.

[19] Zhang L, Lipik V, Miserez A. Complex Coacervates of Oppositely Charged Co-Polypeptides Inspired by the Sandcastle Worm Glue. Journal of Materials Chemistry B. 2016.

[20] Schmitt C, Turgeon SL. Protein/polysaccharide complexes and coacervates in food systems. Advances in Colloid and Interface Science. 2011;167:63-70.

[21] Turgeon SL, Laneuville SI. Protein+ polysaccharide coacervates and complexes: from scientific background to their application as functional ingredients in food products. Modern biopolymer science. 2009:327-63.

[22] Hwang DS, Waite JH, Tirrell M. Promotion of osteoblast proliferation on complex coacervation-based hyaluronic acidrecombinant mussel adhesive protein coatings on titanium. Biomaterials. 2010;31:1080-4.

[23] Yeo Y, Bellas E, Firestone W, Langer R, Kohane DS. Complex coacervates for thermally sensitive controlled release of flavor compounds. Journal of agricultural and food chemistry. 2005;53:7518-25.

[24] de Kruif CG, Weinbreck F, de Vries R. Complex coacervation of proteins and anionic polysaccharides. Current Opinion in Colloid \& Interface Science. 2004;9:340-9.

[25] Bungenberg de Jong H. Complex colloid systems. Colloid science. 1949;2:335-432.

[26] Oparin AI. Origin of Life. Moscow Worker Publisher, Moscow. 1924.

[27] Zhao H, Sun C, Stewart RJ, Waite JH. Cement proteins of the tube-building polychaete Phragmatopoma californica. Journal of biological chemistry. 2005;280:42938-44.

[28] Waite JH, Andersen NH, Jewhurst S, Sun C. Mussel adhesion: finding the tricks worth mimicking. The journal of adhesion. 2005;81:297-317.

[29] Shao H, Bachus KN, Stewart RJ. A Water-Borne Adhesive Modeled after the Sandcastle Glue of P. californica. Macromolecular bioscience. 2009;9:464-71.

[30] Hwang DS, Zeng H, Srivastava A, Krogstad DV, Tirrell M, Israelachvili JN, et al. Viscosity and interfacial properties in a mussel-inspired adhesive coacervate. Soft Matter. 2010;6:3232-6.

[31] Stewart RJ, Weaver JC, Morse DE, Waite JH. The tube cement of Phragmatopoma californica: a solid foam. Journal of Experimental Biology. 2004;207:4727-34. 
[32] Zhao Q, Lee DW, Ahn BK, Seo S, Kaufman Y, Israelachvili JN, et al. Underwater contact adhesion and microarchitecture in polyelectrolyte complexes actuated by solvent exchange. Nature materials. 2016;15:407-12.

[33] Tan Y, Hoon S, Guerette PA, Wei W, Ghadban A, Hao C, et al. Infiltration of chitin by protein coacervates defines the squid beak mechanical gradient. Nature chemical biology. 2015;11:488-95.

[34] Keating CD. Aqueous Phase Separation as a Possible Route to Compartmentalization of Biological Molecules. Accounts of Chemical Research. 2012;45:2114-24.

[35] Brangwynne CP, Eckmann CR, Courson DS, Rybarska A, Hoege C, Gharakhani J, et al. Germline P granules are liquid droplets that localize by controlled dissolution/condensation. Science. 2009;324:1729-32.

[36] Wippich F, Bodenmiller B, Trajkovska Maria G, Wanka S, Aebersold R, Pelkmans L. Dual Specificity Kinase DYRK3 Couples Stress Granule Condensation/Dissolution to mTORC1 Signaling. Cell. 2013;152:791-805.

[37] Berry J, Weber SC, Vaidya N, Haataja M, Brangwynne CP. RNA transcription modulates phase transition-driven nuclear body assembly. Proceedings of the National Academy of Sciences. 2015;112:E5237-E45.

[38] Barbarese E, Koppel DE, Deutscher MP, Smith CL, Ainger K, Morgan F, et al. Protein translation components are colocalized in granules in oligodendrocytes. Journal of Cell Science. 1995;108:2781-90.

[39] Han Tina W, Kato M, Xie S, Wu Leeju C, Mirzaei H, Pei J, et al. Cell-free Formation of RNA Granules: Bound RNAs Identify Features and Components of Cellular Assemblies. Cell.149:768-79.

[40] Kato M, Han Tina W, Xie S, Shi K, Du X, Wu Leeju C, et al. Cell-free Formation of RNA Granules: Low Complexity Sequence Domains Form Dynamic Fibers within Hydrogels. Cell.149:753-67.

[41] Anderson P, Kedersha N. RNA granules. The Journal of Cell Biology. 2006;172:803-8.

[42] Li YR, King OD, Shorter J, Gitler AD. Stress granules as crucibles of ALS pathogenesis. The Journal of Cell Biology. 2013;201:361-72.

[43] Aumiller Jr WM, Keating CD. Phosphorylation-mediated RNA/peptide complex coacervation as a model for intracellular liquid organelles. Nat Chem. 2016;8:129-37.

[44] Lee CF, Brangwynne CP, Gharakhani J, Hyman AA, Jülicher F. Spatial organization of the cell cytoplasm by positiondependent phase separation. Physical review letters. 2013;111:088101.

[45] Brangwynne CP. Phase transitions and size scaling of membrane-less organelles. The Journal of cell biology. 2013;203:87581.

[46] Brangwynne CP, Mitchison TJ, Hyman AA. Active liquid-like behavior of nucleoli determines their size and shape in Xenopus laevis oocytes. Proceedings of the National Academy of Sciences. 2011;108:4334-9.

[47] Lin Y, Protter DS, Rosen MK, Parker R. Formation and maturation of phase-separated liquid droplets by RNA-binding proteins. Molecular cell. 2015;60:208-19.

[48] Li P, Banjade S, Cheng H-C, Kim S, Chen B, Guo L, et al. Phase transitions in the assembly of multivalent signalling proteins. Nature. 2012;483:336-40.

[49] Weber SC, Brangwynne CP. Getting RNA and protein in phase. Cell. 2012;149:1188-91.

[50] Priftis D, Farina R, Tirrell M. Interfacial Energy of Polypeptide Complex Coacervates Measured via Capillary Adhesion†. Langmuir. 2012;28:8721-9.

[51] Lim S, Moon D, Kim HJ, Seo JH, Kang IS, Cha HJ. Interfacial tension of complex coacervated mussel adhesive protein according to the Hofmeister series. Langmuir. 2014;30:1108-15.

[52] Kayitmazer AB, Strand SP, Tribet C, Jaeger W, Dubin PL. Effect of polyelectrolyte structure on protein-polyelectrolyte coacervates: Coacervates of bovine serum albumin with poly (diallyldimethylammonium chloride) versus chitosan.

Biomacromolecules. 2007;8:3568-77.

[53] de Kruif C, Pedersen J, Huppertz T, Anema SG. Coacervates of Lactotransferrin and $\beta$-or $\kappa$-Casein: Structure Determined Using SAXS. Langmuir. 2013;29:10483-90.

[54] Huang K-Y, Yoo HY, Jho Y, Han S, Hwang DS. Bicontinuous Fluid Structure with Low Cohesive Energy: Molecular Basis for Exceptionally Low Interfacial Tension of Complex Coacervate Fluids. ACS Nano. 2016.

[55] Kizilay E, Kayitmazer AB, Dubin PL. Complexation and coacervation of polyelectrolytes with oppositely charged colloids. Advances in colloid and interface science. 2011;167:24-37.

[56] van der Burgh S, de Keizer A, Cohen Stuart MA. Complex Coacervation Core Micelles. Colloidal Stability and Aggregation Mechanism. Langmuir. 2004;20:1073-84.

[57] Voets IK, de Keizer A, Cohen Stuart MA. Complex coacervate core micelles. Advances in Colloid and Interface Science. 2009;147-148:300-18.

[58] Menger FM, Peresypkin AV. A combinatorially-derived structural phase diagram for 42 zwitterionic geminis. Journal of the American Chemical Society. 2001;123:5614-5.

[59] Menger F, Peresypkin A, Caran K, Apkarian R. A sponge morphology in an elementary coacervate. Langmuir. 2000;16:9113-6.

[60] Menger FM, Seredyuk VA, Apkarian RP, Wright ER. Colloidal assemblies of branched geminis studied by cryo-etchHRSEM. Journal of the American Chemical Society. 2002;124:12408-9.

[61] Imura T, Yanagishita H, Kitamoto D. Coacervate formation from natural glycolipid: one acetyl group on the headgroup triggers coacervate-to-vesicle transition. Journal of the American Chemical Society. 2004;126:10804-5.

[62] Peresypkin AV, Menger FM. Zwitterionic geminis. Coacervate formation from a single organic compound. Organic Letters. 1999;1:1347-50. 
[63] Ducel V, Richard J, Popineau Y, Boury F. Rheological interfacial properties of plant protein-arabic gum coacervates at the oil-water interface. Biomacromolecules. 2005;6:790-6.

[64] Pallas NR, Harrison Y. Selected Papers from a Symposium on Recent Progress in Interfacial Tensiometry, held at the Third Chemical Congress of North AmericaAn automated drop shape apparatus and the surface tension of pure water. Colloids and Surfaces. 1990;43:169-94.

[65] Arneodo C, Baszkin A, Benoit JP, Thies C. Interfacial Tension Behavior of Citrus Oils Against Phases Formed by Complex Coacervation of Gelatin. Flavor Encapsulation. Vol. 370: American Chemical Society; 1988. Chapter 15. p. 132-47.

[66] Priftis D, Farina R, Tirrell M. Interfacial Energy of Polypeptide Complex Coacervates Measured via Capillary Adhesion. Langmuir. 2012;28:8721-9.

[67] Spruijt E, Sprakel J, Stuart MAC, van der Gucht J. Interfacial tension between a complex coacervate phase and its coexisting aqueous phase. Soft Matter. 2010;6:172-8.

[68] De Ruiter L. Bungendberg de Jong. HB Proc Sect Sci, Koninklijke Nederlandse Akademie van Wetenschappen. 1947;50:836-48.

[69] Riggleman RA, Kumar R, Fredrickson GH. Investigation of the interfacial tension of complex coacervates using fieldtheoretic simulations. The Journal of chemical physics. 2012;136:024903.

[70] Qin J, Priftis D, Farina R, Perry SL, Leon L, Whitmer J, et al. Interfacial tension of polyelectrolyte complex coacervate phases. ACS Macro Letters. 2014;3:565-8.

[71] Perry SL, Li Y, Priftis D, Leon L, Tirrell M. The effect of salt on the complex coacervation of vinyl polyelectrolytes. Polymers. 2014;6:1756-72.

[72] Spruijt E, Sprakel J, Lemmers M, Stuart MAC, van der Gucht J. Relaxation Dynamics at Different Time Scales in Electrostatic Complexes: Time-Salt Superposition. Physical Review Letters. 2010;105:208301.

[73] Armstrong BD, Han S. Overhauser dynamic nuclear polarization to study local water dynamics. Journal of the American Chemical Society. 2009;131:4641-7.

[74] Kausik R, Srivastava A, Korevaar PA, Stucky G, Waite JH, Han S. Local Water Dynamics in Coacervated Polyelectrolytes Monitored through Dynamic Nuclear Polarization-Enhanced 1H NMR. Macromolecules. 2009;42:7404-12.

[75] Ortony JH, Hwang DS, Franck JM, Waite JH, Han S. Asymmetric Collapse in Biomimetic Complex Coacervates Revealed by Local Polymer and Water Dynamics. Biomacromolecules. 2013;14:1395-402.

[76] Andreas J, Hauser E, Tucker W. Boundary tension by pendant drops1. The Journal of Physical Chemistry. 1938;42:1001-19.

[77] Bashforth F, Adams JC. An attempt to test the theories of capillary action: by comparing the theoretical and measured forms of drops of fluid. With an explanation of the method of integration employed in constucting the tables which give the theoretical forms of such drops: University Press; 1883.

[78] Ducker WA, Senden TJ, Pashley RM. Direct measurement of colloidal forces using an atomic force microscope. 1991.

[79] Butt H-J. Measuring electrostatic, van der Waals, and hydration forces in electrolyte solutions with an atomic force microscope. Biophysical Journal. 1991;60:1438.

[80] Sprakel J, Besseling N, Leermakers F, Stuart MC. Equilibrium capillary forces with atomic force microscopy. Physical review letters. 2007;99:104504.

[81] Hofmeister F. Zur lehre von der wirkung der salze. Naunyn-Schmiedeberg's Archives of Pharmacology. 1888;25:1-30.

[82] Israelachvili JN. Intermolecular and surface forces: revised third edition: Academic press; 2011.

[83] Miller DR, Das S, Huang K-Y, Han S, Israelachvili JN, Waite JH. Mussel Coating Protein-Derived Complex Coacervates

Mitigate Frictional Surface Damage. ACS Biomaterials Science \& Engineering. 2015;1:1121-8.

[84] Michaeli I, Overbeek JTG, Voorn M. Phase separation of polyelectrolyte solutions. Journal of Polymer Science.

1957;23:443-50.

[85] Cahn JW, Hilliard JE. Free Energy of a Nonuniform System. I. Interfacial Free Energy. The Journal of Chemical Physics. 1958;28:258-67.

[86] Borue VY, Erukhimovich IY. A statistical theory of globular polyelectrolyte complexes. Macromolecules. 1990;23:3625-32.

[87] Lifshitz IM, Grosberg AY, Khokhlov AR. Some problems of the statistical physics of polymer chains with volume interaction. Reviews of Modern Physics. 1978;50:683-713.

[88] Fredrickson G. The equilibrium theory of inhomogeneous polymers. 2006.

[89] Lee J, Popov YO, Fredrickson GH. Complex coacervation: A field theoretic simulation study of polyelectrolyte complexation. The Journal of chemical physics. 2008;128:224908.

[90] Wang Z-G. Fluctuation in electrolyte solutions: The self energy. Physical Review E. 2010;81:021501.

[91] Marcus Y. Effect of Ions on the Structure of Water: Structure Making and Breaking. Chemical Reviews. 2009;109:1346-70.

[92] Moreira AG, Netz RR. Strong-coupling theory for counter-ion distributions. EPL (Europhysics Letters). 2000;52:705.

[93] Alexander S, Chaikin PM, Grant P, Morales GJ, Pincus P, Hone D. Charge renormalization, osmotic pressure, and bulk modulus of colloidal crystals: Theory. The Journal of Chemical Physics. 1984;80:5776-81.

[94] Manning GS. Limiting Laws and Counterion Condensation in Polyelectrolyte Solutions I. Colligative Properties. The Journal of Chemical Physics. 1969;51:924-33.

[95] Perry SL, Sing CE. PRISM-Based Theory of Complex Coacervation: Excluded Volume versus Chain Correlation. Macromolecules. 2015;48:5040-53.

[96] Dutta S, Jho YS. Shell formation in short like-charged polyelectrolytes in a harmonic trap. Physical Review E. 2016;93:012503. 
[97] Spruijt E, Westphal AH, Borst JW, Cohen Stuart MA, van der Gucht J. Binodal compositions of polyelectrolyte complexes. Macromolecules. 2010;43:6476-84.

[98] Spruijt E, Leermakers FAM, Fokkink R, Schweins R, van Well AA, Cohen Stuart MA, et al. Structure and Dynamics of Polyelectrolyte Complex Coacervates Studied by Scattering of Neutrons, X-rays, and Light. Macromolecules. 2013;46:4596-605. [99] Du X, Seeman D, Dubin PL, Hoagland DA. Nonfreezing Water Structuration in Heteroprotein Coacervates. Langmuir. 2015;31:8661-6.

[100] Nagura M, Konishi J, MON Y, Ishikawa H. Fine-structure of coacervates prepared from aqueous-solutions of denatured tropocollagen and of chondoroitin sulfate-C. Kobunshi Ronbunshu. 1988;45:581-6.

[101] Zeng H, Hwang DS, Israelachvili JN, Waite JH. Strong reversible Fe3+-mediated bridging between dopa-containing protein films in water. Proceedings of the National Academy of Sciences. 2010;107:12850-3.

[102] Lu Q, Danner E, Waite JH, Israelachvili JN, Zeng H, Hwang DS. Adhesion of mussel foot proteins to different substrate surfaces. Journal of The Royal Society Interface. 2013;10:20120759.

[103] Hwang DS, Waite JH. Three intrinsically unstructured mussel adhesive proteins, mfp-1, mfp-2, and mfp-3: Analysis by circular dichroism. Protein Science. 2012;21:1689-95.

[104] Ortony JH, Choi S-H, Spruell JM, Hunt JN, Lynd NA, Krogstad DV, et al. Fluidity and water in nanoscale domains define coacervate hydrogels. Chemical Science. 2014;5:58-67.

[105] Jeschke G. Determination of the nanostructure of polymer materials by electron paramagnetic resonance spectroscopy. Macromolecular rapid communications. 2002;23:227-46.

[106] Jeschke G, Polyhach Y. Distance measurements on spin-labelled biomacromolecules by pulsed electron paramagnetic resonance. Physical Chemistry Chemical Physics. 2007;9:1895-910.

[107] Hinderberger D. EPR Spectroscopy in Polymer Science. EPR Spectroscopy: Springer; 2011. p. 67-89.

[108] Xia Y, Li Y, Burts AO, Ottaviani MF, Tirrell DA, Johnson JA, et al. EPR study of spin labeled brush polymers in organic solvents. Journal of the American Chemical Society. 2011;133:19953-9.

[109] Ortony JH, Newcomb CJ, Matson JB, Palmer LC, Doan PE, Hoffman BM, et al. Internal dynamics of a supramolecular nanofibre. Nature materials. 2014;13:812-6.

[110] Huang K-Y, Kingsley CN, Sheil R, Cheng C-Y, Bierma JC, Roskamp KW, et al. Stability of protein-specific hydration shell on crowding. Journal of the American Chemical Society. 2016;138:5392-402.

[111] Franck JM, Pavlova A, Scott JA, Han S. Quantitative cw Overhauser effect dynamic nuclear polarization for the analysis of local water dynamics. Progress in nuclear magnetic resonance spectroscopy. 2013;74:33-56.

[112] Stals PJ, Cheng C-Y, van Beek L, Wauters AC, Palmans AR, Han S, et al. Surface water retardation around single-chain polymeric nanoparticles: critical for catalytic function? Chemical Science. 2016.

[113] Song J, Allison B, Han S. Local water diffusivity as a molecular probe of surface hydrophilicity. MRS Bulletin.

2014;39:1082-8.

[114] Singh SS, Aswal V, Bohidar H. Structural studies of agar-gelatin complex coacervates by small angle neutron scattering, rheology and differential scanning calorimetry. International journal of biological macromolecules. 2007;41:301-7.

[115] Wu J, Lin W, Wang Z, Chen S, Chang Y. Investigation of the hydration of nonfouling material poly (sulfobetaine methacrylate) by low-field nuclear magnetic resonance. Langmuir. 2012;28:7436-41.

[116] Kizilay E, Dinsmore AD, Hoagland DA, Sun L, Dubin PL. Evolution of hierarchical structures in polyelectrolyte-micelle coacervates. Soft Matter. 2013;9:7320-32.

[117] Gummel J, Cousin F, Verbavatz J-M, Boué F. Wide scale range structure in polyelectrolyte-protein dense complexes: Where Sans meets freeze-fracture microscopy. The Journal of Physical Chemistry B. 2007;111:8540-6.

[118] Liberatore MW, Wyatt NB, Henry M, Dubin PL, Foun E. Shear-induced phase separation in polyelectrolyte/mixed micelle coacervates. Langmuir. 2009;25:13376-83.

[119] Wang X, Li Y, Wang Y-W, Lal J, Huang Q. Microstructure of $\beta$-lactoglobulin/pectin coacervates studied by small-angle neutron scattering. The Journal of Physical Chemistry B. 2007;111:515-20.

[120] Krogstad DV, Choi S-H, Lynd NA, Audus DJ, Perry SL, Gopez JD, et al. Small Angle Neutron Scattering Study of Complex Coacervate Micelles and Hydrogels Formed from Ionic Diblock and Triblock Copolymers. The Journal of Physical Chemistry B. 2014;118:13011-8.

[121] Horkay F, Hecht A-M, Geissler E. Similarities between polyelectrolyte gels and biopolymer solutions. Journal of Polymer Science Part B: Polymer Physics. 2006;44:3679-86.

[122] Chodankar S, Aswal VK, Kohlbrecher J, Vavrin R, Wagh AG. Structural study of coacervation in protein-polyelectrolyte complexes. Physical Review E. 2008;78:031913.

[123] Singh SS, Aswal VK, Bohidar HB. Structural studies of agar-gelatin complex coacervates by small angle neutron scattering, rheology and differential scanning calorimetry. International Journal of Biological Macromolecules. 2007;41:301-7. [124] Weinbreck F, Tromp RH, de Kruif CG. Composition and Structure of Whey Protein/Gum Arabic Coacervates.

Biomacromolecules. 2004;5:1437-45. 


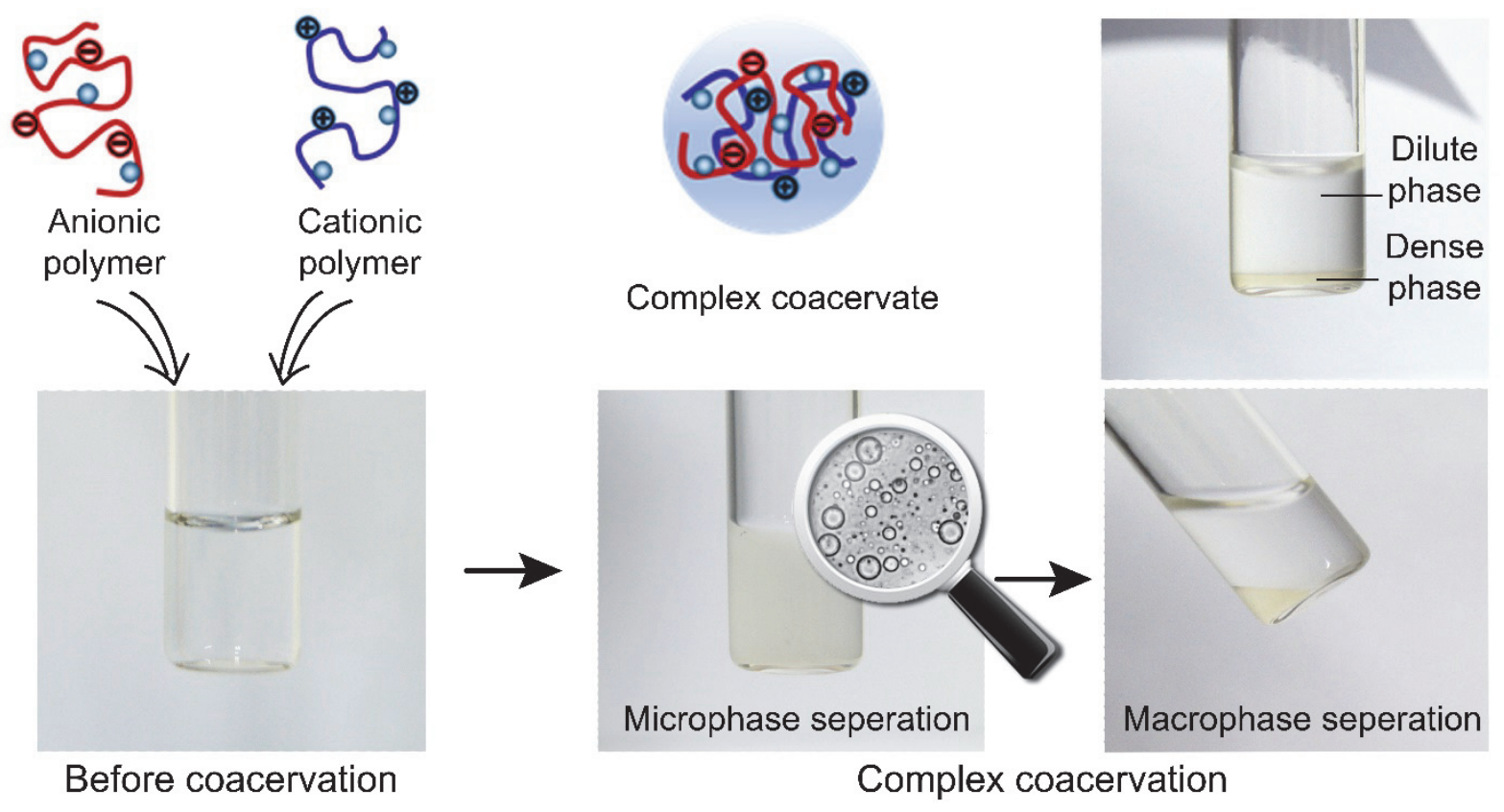

Figure 1. Schematics of complex coacervation of anionic polymer (hyaluronic acid, HA) and cationic polymer (chitosan) in $0.1 \mathrm{M}$ sodium acetate at $\mathrm{pH} 5.0$ 


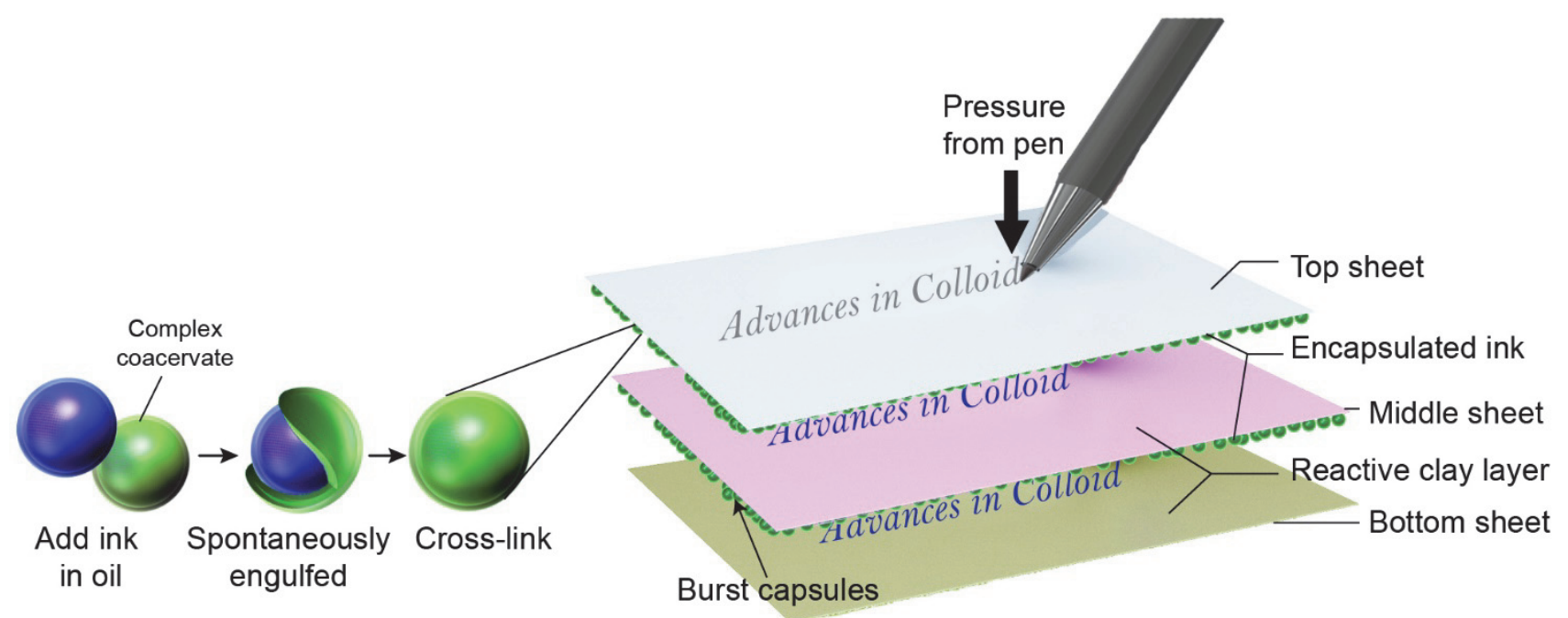

Figure 2. Schematics of the first commercial example of an application of the coacervate phase for encapsulation. This encapsulated ink oil is stabilized by a cross-linker, and the microcapsules of ink oil is utilized for generating "carbonless copying paper".Complex Coacervate Fluids. ACS Nano. 2016. 


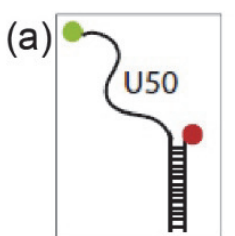

(b)

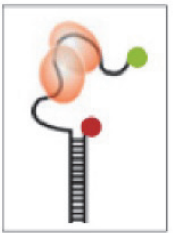

RNA-FRET LAF-1 FRET
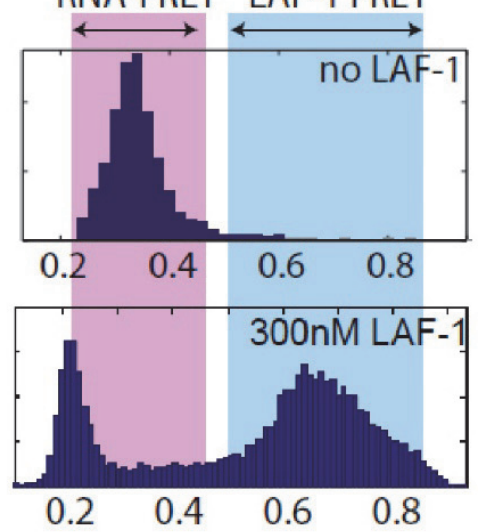

FRET
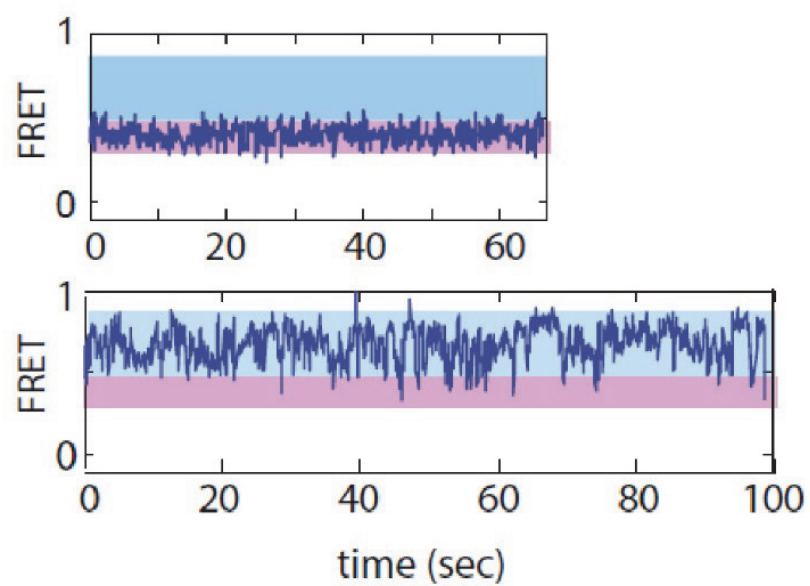

Figure 3. FRET fluctuation of LAF-1-RNA droplets represented by FRET histogram and single molecule FRET trace. (a) FRET of polyU50 RNA before LAF-1 binding. (b) Additional broad FRET peak emerges with clear separation between two FRET ranges, suggesting altered interactions between LAF-1 and the RNA substrate. FRET fluctuations on increased LAF-1 concentration indicate enhanced dynamics between LAF-1 and RNA. Similar pattern was observed when approaching the phase boundary by changing salt concentration with fixed LAF1 concentration.

Source: Figure 3 was taken from Elbaum-Garfinkle S, Kim Y, Szczepaniak K, Chen CC-H, Eckmann CR, Myong S, et al. The disordered P granule protein LAF-1 drives phase separation into droplets with tunable viscosity and dynamics. Proceedings of the National Academy of Sciences. 2015;112:7189-94. 
(a)

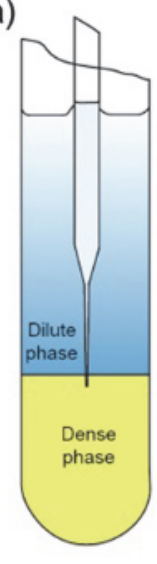

(c)

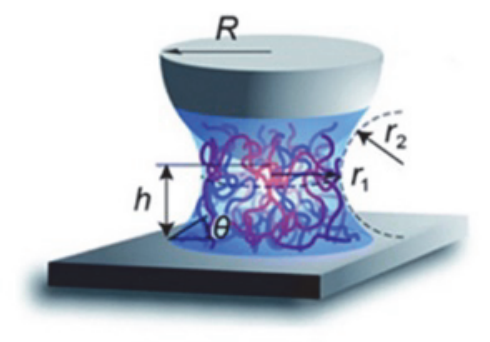

(b)

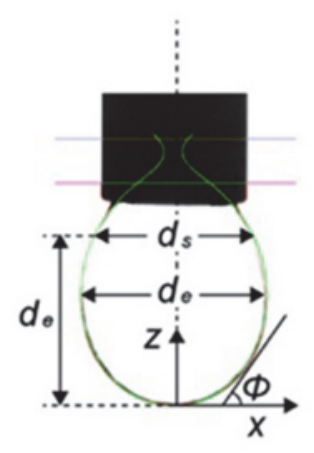

(d)

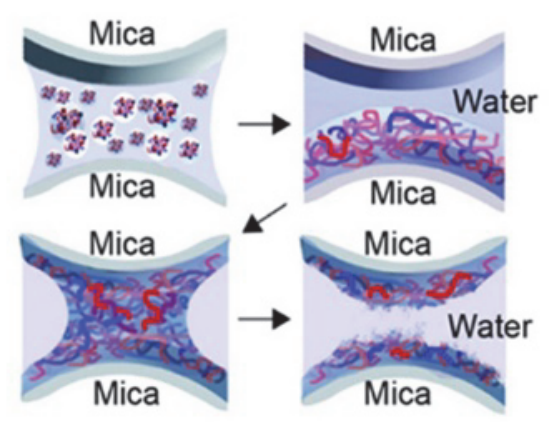

Figure 4. Four types of experimental methods have been utilized for measuring the interfacial tension of the coacervate. (a) Modified capillary rise method proposed by De Ruiter and Bungenberg-de Jong (b) Pendant drop method (c) Colloidal probe atomic force microscopy (CPAFM) (d) Surface forces apparatus (SFA)

Source: Figure 4 (c) was taken from Spruijt E, Sprakel J, Stuart MAC, van der Gucht J. Interfacial tension between a complex coacervate phase and its coexisting aqueous phase. Soft Matter. 2010;6:172-8. 
(a)

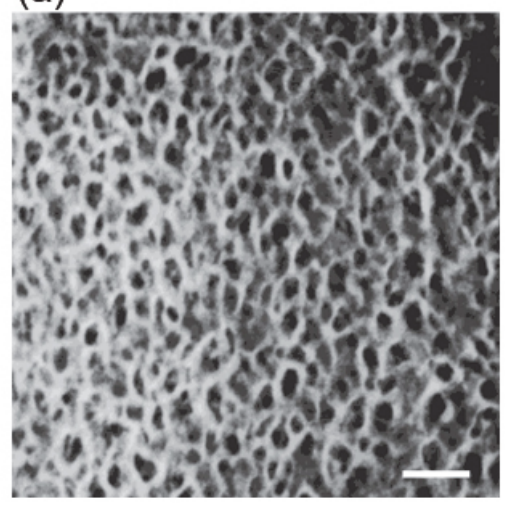

(b)

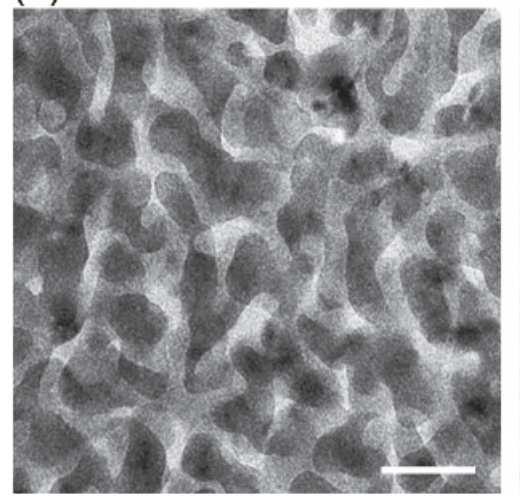

(c)

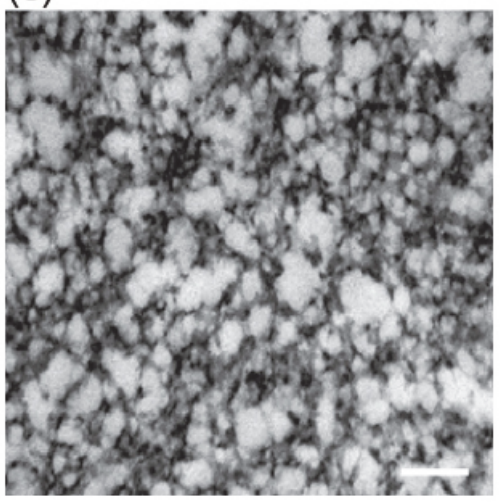

Figure 5. Imaging of bicontinuous phase structure with different imaging techniques. (a) Higher magnification cryo-Scanning electron microscopy reveals the sponge like structure of the coacervate surface (bar $-=667 \mathrm{~nm}$ ) (b) cryo-transmission electron microscopy of coacervate. The scale bar is $100 \mathrm{~nm}$. (c) High pressure cryo-transmission electron microscopy of coacervate. The scale bar is $500 \mathrm{~nm}$.

Source: Figure 5 (a) was taken from Menger F, Peresypkin A, Caran K, Apkarian R. A sponge morphology in an elementary coacervate. Langmuir. 2000;16:9113-6.

Figure 5 (b) was taken from Hwang DS, Zeng H, Srivastava A, Krogstad DV, Tirrell M, Israelachvili $\mathrm{JN}$, et al. Viscosity and interfacial properties in a mussel-inspired adhesive coacervate. Soft Matter. 2010;6:3232-6.

Figure 5 (c) was taken from Huang K-Y, Yoo HY, Jho Y, Han S, Hwang DS. Bicontinuous Fluid Structure with Low Cohesive Energy: Molecular Basis for Exceptionally Low Interfacial Tension of Complex Coacervate Fluids. ACS Nano. 2016;10;5051-62 
(a)
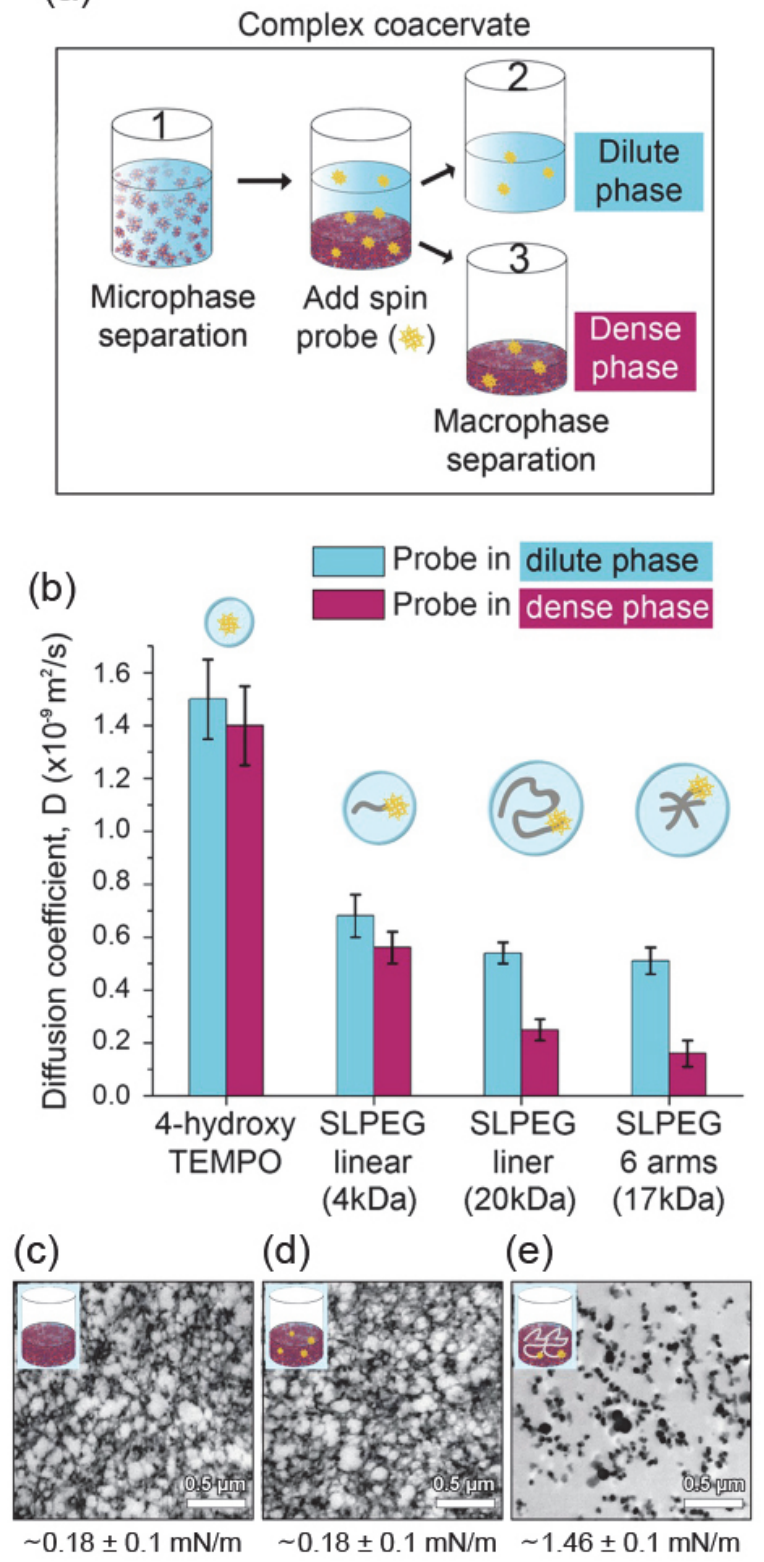

Figure 6. (a) Schematics of the spin probe added complex coacervation of mfp-1 and hyaluronic acid (HA) and (b) translational diffusion coefficients of water in the dilute and dense phases of complex coacervates (light blue and purple, respectively) by ODNP using different size spin probes. (c-e) Structures of coacervated mfp-1/HA under a transmission electron microscope when (c) mfp-1 and HA were added at an optimal ratio and salt condition, (d) optimal condition of mfp-1 and HA were added with $120 \mathrm{mM}$ free spin probe 4-hydroxy TEMPO and (e) optimal condition of mfp-1 and HA were added with $\sim 5 \%$ 20kDa PEG tethered spin probe. (c), (d) a low interfacial tension of $\sim 0.18 \pm 0.1 \mathrm{mN} / \mathrm{m}$, (e) significant increase in interfacial tension to $1.46 \pm 0.1 \mathrm{mN} / \mathrm{m}$ when only $250 \mu \mathrm{M}$ of PEG is added to the coacervate fluid. 
Source: Figure 6 was taken from Huang K-Y, Yoo HY, Jho Y, Han S, Hwang DS. Bicontinuous Fluid Structure with Low Cohesive Energy: Molecular Basis for Exceptionally Low Interfacial Tension of Complex Coacervate Fluids. ACS Nano. 2016;10;5051-62 


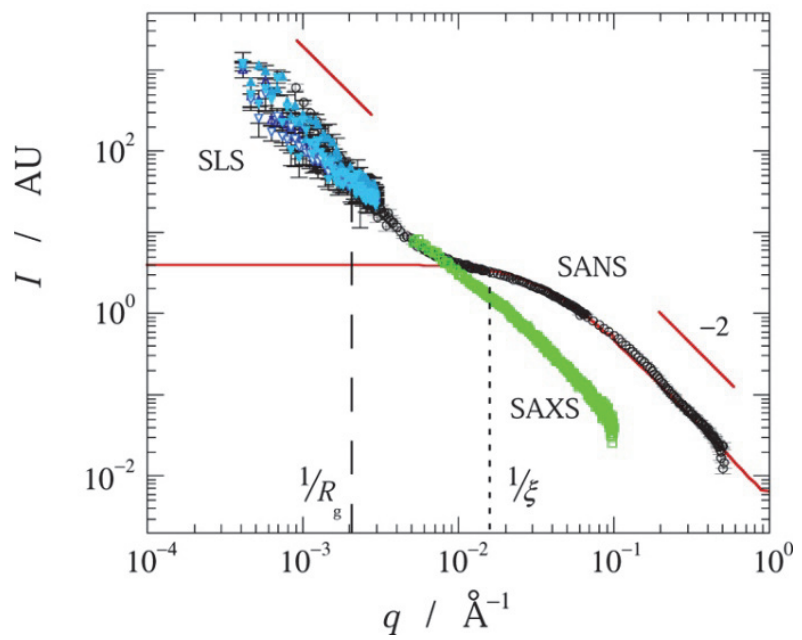

Figure 7. Combination of SANS, SAXS and SLS curves for PDMAEMA $574 / \mathrm{PAA}_{550}$ complex coacervates at salt concentration of $1.2 \mathrm{M}$. All the three scattering methods shows an upturn of intensity at the low $q$ value, which agrees with previous studies on semi-dilute systems. The solid line is an ideal model of semi-diluted polymer solution fitting the SANS data. The labels $1 / R_{\mathrm{g}}$ and $1 / \xi$ indicate the approximate inverse radius of gyration and inverse mesh size of the polyelectrolytes and complex coacervates, respectively.

Source: Figure 7 was taken from Spruijt E, Leermakers FAM, Fokkink R, Schweins R, van Well AA, Cohen Stuart MA, et al. Structure and Dynamics of Polyelectrolyte Complex Coacervates Studied 

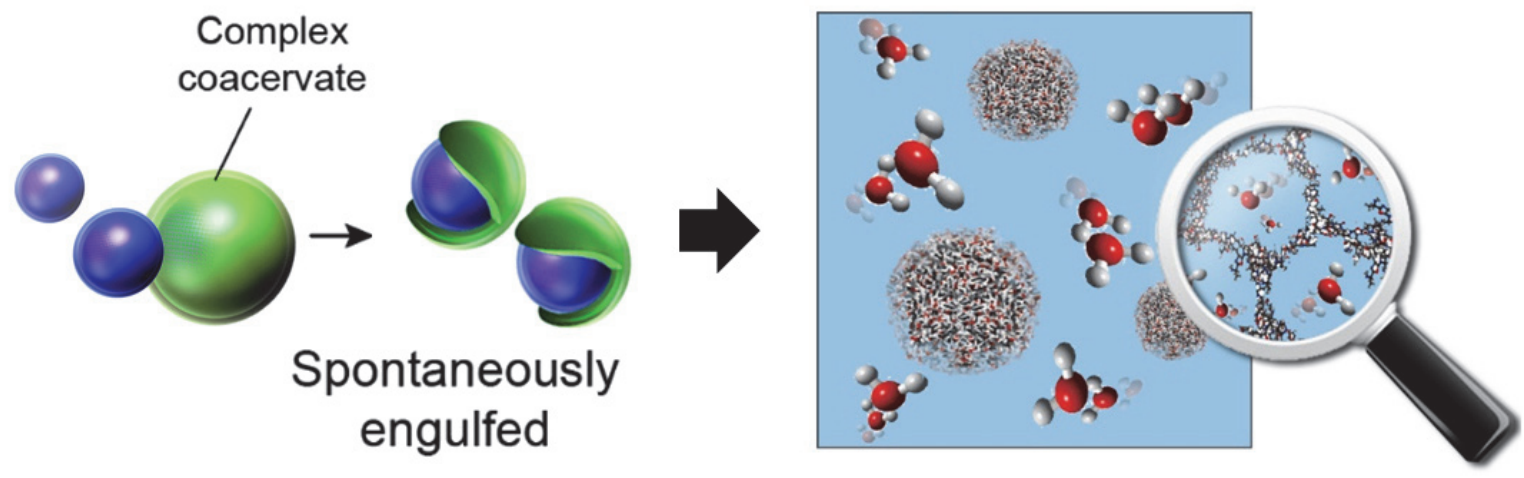